\title{
Single-cell transcriptome profiling of an adult human cell atlas of 15 major organs
}

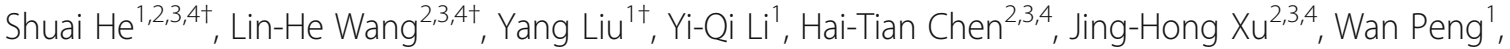 \\ Guo-Wang Lin ${ }^{1,5}$, Pan-Pan Wei ${ }^{1}$, Bo Li ${ }^{6,7}$, Xiaojun Xia ${ }^{1}$, Dan Wang ${ }^{1}$, Jin-Xin Bei ${ }^{1,8^{*}}$ D. Xiaoshun He $\mathrm{e}^{2,3,4^{*}}$ and \\ Zhiyong Guo 2,3,4*
}

\author{
* Correspondence: beijx@sysucc.org. \\ cn; gdtrc@163.com; rockyucsf1981@ \\ 126.com \\ †Shuai He, Lin-He Wang and Yang \\ Liu contributed equally to this work. \\ ${ }^{1}$ Sun Yat-sen University Cancer \\ Center, State Key Laboratory of \\ Oncology in South China, \\ Collaborative Innovation Center for \\ Cancer Medicine, Guangzhou \\ 510060, People's Republic of China \\ ${ }^{2}$ Organ Transplant Center, The First \\ Affiliated Hospital, Sun Yat-sen \\ University, Guangzhou 510080, \\ People's Republic of China \\ Full list of author information is \\ available at the end of the article
}

\begin{abstract}
Background: As core units of organ tissues, cells of various types play their harmonious rhythms to maintain the homeostasis of the human body. It is essential to identify the characteristics of cells in human organs and their regulatory networks for understanding the biological mechanisms related to health and disease. However, a systematic and comprehensive single-cell transcriptional profile across multiple organs of a normal human adult is missing.

Results: We perform single-cell transcriptomes of 84,363 cells derived from 15 tissue organs of one adult donor and generate an adult human cell atlas. The adult human cell atlas depicts 252 subtypes of cells, including major cell types such as T, B, myeloid, epithelial, and stromal cells, as well as novel $\mathrm{COCH}^{+}$fibroblasts and FibSmo cells, each of which is distinguished by multiple marker genes and transcriptional profiles. These collectively contribute to the heterogeneity of major human organs. Moreover, T cell and $\mathrm{B}$ cell receptor repertoire comparisons and trajectory analyses reveal direct clonal sharing of $T$ and $B$ cells with various developmental states among different tissues. Furthermore, novel cell markers, transcription factors, and ligand-receptor pairs are identified with potential functional regulations in maintaining the homeostasis of human cells among tissues.
\end{abstract}

Conclusions: The adult human cell atlas reveals the inter- and intra-organ heterogeneity of cell characteristics and provides a useful resource in uncovering key events during the development of human diseases in the context of the heterogeneity of cells and organs.

Keywords: Human cell atlas, Single-cell RNA sequencing, TCR, BCR, Transcriptome

\section{Introduction}

The human body consists of multiple organs, where multiple types of cells are the core units of structure and function. Like instruments from different families in a symphony orchestra, cells and organs play their harmonious rhythms to maintain the homeostasis of the human body. Yet, perturbations in the homeostasis lead to various pathological conditions. Therefore, it is essential to identify characteristics of the cells in human

(c) The Author(s). 2020 Open Access This article is licensed under a Creative Commons Attribution 4.0 International License, which permits use, sharing, adaptation, distribution and reproduction in any medium or format, as long as you give appropriate credit to the original author(s) and the source, provide a link to the Creative Commons licence, and indicate if changes were made. The images or other third party material in this article are included in the article's Creative Commons licence, unless indicated otherwise in a credit line to the material. If material is not included in the article's Creative Commons licence and your intended use is not permitted by statutory regulation or exceeds the permitted use, you will need to obtain permission directly from the copyright holder. To view a copy of this licence, visit http://creativecommons.org/licenses/by/4.0/. The Creative Commons Public Domain Dedication waiver (http://creativecommons.org/publicdomain/zero/1.0/) applies to the data made available in this article, unless otherwise stated in a credit line to the data. 
organs and their regulatory networks for understanding the biological mechanisms related to health and disease.

Recent technological innovations in transcriptional profiling using single-cell RNA sequencing (scRNA-seq) have provided a promising strategy to quantify gene expression at the genome-wide level in thousands of individual cells simultaneously [1-3]. This has expanded our knowledge regarding cellular heterogeneity and networks, as well as our understanding in developments in human tissues and organs at the single-cell resolution [4-11]. Previous studies have demonstrated the cell composition for many human and mice tissues, including the brain [12], kidneys [13], lungs [14], and skin [15]. The strategy also empowers the identification of novel cell types. Cells marked by cystic fibrosis transmembrane conductance regulator $(C F T R)$ were identified in the lungs of human and mouse and were able to regulate luminal $\mathrm{pH}$ that was implicated in the pathogenesis of cystic fibrosis [16]. Non-genetic cellular heterogeneity has been revealed in hematopoietic progenitor cells and keratinocytes, which play important roles in maintaining hematopoiesis [17] and compartmentalizing crucial molecular activities in human epidermis [15], respectively. For the development of human embryos, transcriptome analyses of about 70,000 single cells from the first trimester's placenta with matched maternal blood and decidual cells uncover the cellular organization of decidua and placenta, as well as distinctive immunomodulatory and chemokine profiles of decidual natural killer (NK) cells [18]. In addition, the single-cell transcriptional profiles of embryonic and adult organs in mice have been reported, which reveal the landscape of organogenesis and the cellular heterogeneity in organs $[8,9,19]$. A very recent study on major human cell types using multiple organs from different donors revealed the genetic regulation for fetal-to-adult cell-type transitions and genetic conservation in mammalian cells [20]. However, a systematic and comprehensive single-cell transcriptional profile of multiple organs from a normal human adult has been pending. Previous studies with scRNA-seq on human samples were mostly restricted to a few specific organs with disease conditions, and they did not attempt to characterize the heterogeneity and connections among multiple organs in a same individual.

Here, we aimed to investigate the transcriptional heterogeneity and interactions of cells from an adult human's organs at the single-cell resolution level. Using scRNA-seq, we profiled the transcriptomes of more than 84,000 cells of 15 organs from one individual donor. Comprehensive comparisons within and across tissues for distinct cell types were performed to reveal the intercellular complexity of gene profiles, active transcription factors, and potential biological functions, as well as potential inter-cell connections. The resulting high-resolution adult human cell atlas (AHCA) provides a global view of various cell populations and connections in the human body and is also a useful resource to investigate the biology of normal human cells and the development of diseases affecting different organs.

\section{Results}

\section{Global view of single-cell RNA sequencing of 15 organ samples}

Viable single cells were prepared from the tissue samples of 15 different organs of a research-consented adult donor (Fig. 1a). mRNA transcripts from each sample were ligated with barcoded indexes at $5^{\prime}$-end and reverse transcribed into cDNA, using 


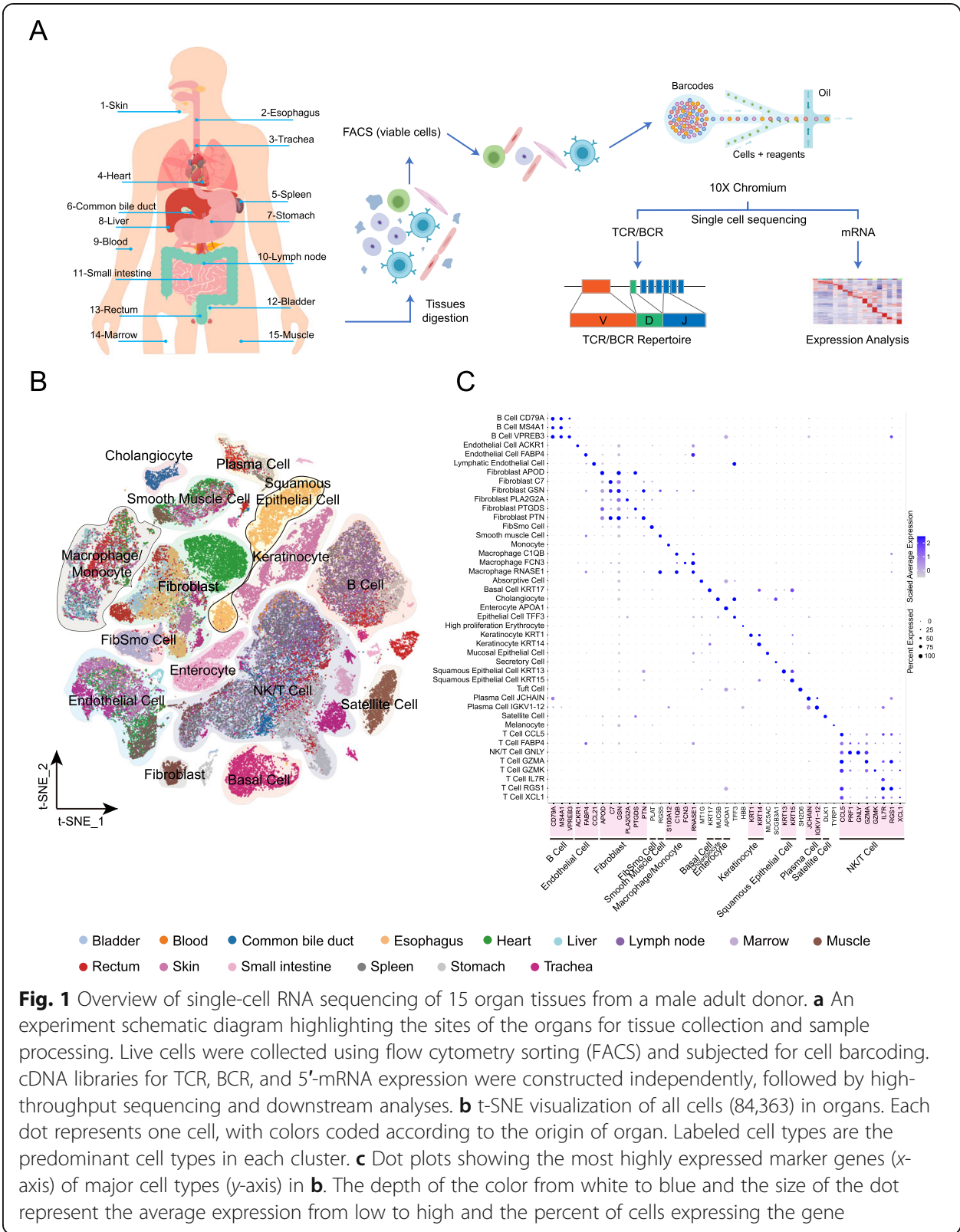

GemCode technology (10x Genomics, USA). cDNA libraries including enriched fragments spanning the full-length V(D)J segments of T cell receptors (TCR) or B cell receptors (BCR), and 5 '-end fragments for gene expression were separately constructed, which were subsequently subjected for high-throughput sequencing. On average, we obtained more than 400 million sequencing reads for each organ sample, which resulted in a median sequencing saturation (covering the fraction of library complexity) of $88 \%$ (61.6-97\%) for each sample (Additional file 1: Figure S1A and Additional file 2: Table S1). After primary quality control (QC) filters, 91,393 cells were identified (Additional file 1: Figure S1B and Additional file 2: Table S2). Higher number of UMIs and more transcribed genes were observed in the skin and trachea samples (with median UMIs of 4022.5 and 4100.5 and genes of 1528 and 1653, respectively) compared with the other organs (Additional file 1: Figure S1C, D, and Additional file 2: Table S2). We 
obtained 66,225 sequencing read pairs for each cell and 6093 cells for each organ on average (Additional file 2: Table S1, S2), with more than 2.4 and 3.5 times deeper sequencing of median genes and UMIs than a recent study [20]. The cells in each organ were classified using unsupervised clustering, and cell types were assigned based on canonical marker genes (Additional file 2: Table S3). Next, visualization of the cells by $\mathrm{t}$-distributed stochastic neighbor embedding ( $t$-SNE) revealed multiple subpopulations of cells in each organ, with the numbers of clusters ranging from 9 in the blood to 25 in the skin (Additional file 1: Figure S2, Figure S2 continued, and Additional file 2: Table S4-S18). Clusters due to cell doublets were identified and excluded for each organ, which resulted in a total of 84,363 cells for the downstream analyses (Additional file 2: Table S2).

With transcriptional profiles of such large number of cells, we identified some rare and novel cell populations. A group of Langerhans cells were identified in the skin sample (1\% of all skin cells) with specific expression of CD207 and CD1A [21]. An even smaller group of 26 sweat gland epithelial cells $(0.31 \%)$ were also identified in the skin sample, which had specific expression of DCD, SCGB2A2, KRT19, MUCL1, and PIP genes (Additional file 1: Figure S3A and Additional file 2: Table S14). A novel group of fibroblasts $(0.43 \%)$ with exclusive expression of $\mathrm{COCH}$ were identified in the skin (Additional file 1: Figure S2 continued, Figure S3A, and Additional file 2: Table S14). Of note, another novel group of cells were assigned as FibSmo with a co-expression of MMP2 and ACTA2, which are marker genes for fibroblasts and smooth muscle cells, and were identified with higher proportions in the rectum (6.66\%), bladder (17.59\%), and heart $(7.75 \%)$ than in the other tissue organs (Additional file 1: Figure S2, Figure S2 continued, Figure S3B, and Additional file 2: Table S4, S13). Moreover, in contrast to the broad distribution of FibSmo cells in multiple organs, $\mathrm{COCH}^{+}$fibroblasts were identified in limited organs with low abundance, while sweat gland epithelial cells were found specifically in the skin (Additional file 1: Figure S3B). Furthermore, the presence of sweat gland epithelial cells, $\mathrm{COCH}^{+}$fibroblasts, and FibSmo cells was confirmed in multiple tissue samples from independent donors using existing datasets and multiplex immunofluorescence staining assays (Additional file 1: Figure S4-S10 and Additional file 3: Supplementary Notes).

We combined all the 84,363 cells in the cluster analysis and identified 43 clusters in 15 organs (Fig. 1b, Additional file 1: Figure S11 and Additional file 2: Table S19). We observed close clustering of cells from different organs (more than seven organs) for major cell types, including T, B, plasma, endothelial, and smooth muscle cells, as well as fibroblasts, macrophages, and monocytes (Fig. 1b, c, Additional file 1: Figure S11, Additional file 1: Figure S12, and Additional file 2: Table S20). This is consistent with the understanding that cells derived from the same lineage are widely distributed within the human body, especially circulating immune cells. Moreover, multiple clusters were further identified for several major cell types ( $\mathrm{T}$ cells, B cells, fibroblasts, myeloid cells, and endothelial cells), reflecting their heterogeneous transcriptional profiles (Fig. 1c, Additional file 2: Table S19).

The heterogeneity of $\mathrm{T}$ cells in developmental state and clonalities around the body We identified a total of 20,034 T cells prevailing in the immune cells of most organ tissues (Additional file 4: Table S21), which is consistent with a previous finding [22]. 
These included $1472 \gamma \delta$ and 18,292 $\alpha \beta$ T cells. The latter were divided into CD4 ${ }^{+}$ (7006) and $\mathrm{CD}^{+}(11,286) \mathrm{T}$ cells according to their gene profiles and were further grouped into 11 and 21 major unsupervised clusters, respectively (Fig. 2a, b), including naïve/central memory $\mathrm{T}\left(\mathrm{T}_{\mathrm{N} / \mathrm{CM}}\right)$, effector memory $\mathrm{T}\left(\mathrm{T}_{\mathrm{EM}}\right)$, regulatory $\mathrm{T}\left(\mathrm{T}_{\text {reg }}\right)$, tissueresident memory $\mathrm{T}\left(\mathrm{T}_{\mathrm{RM}}\right)$, effector $\mathrm{T}\left(\mathrm{T}_{\mathrm{h} 1}\right.$ for $\mathrm{CD} 4^{+}$and $\mathrm{T}_{\mathrm{EFF}}$ for $\mathrm{CD} 8^{+} \mathrm{T}$ cell), intraepithelial lymphocyte (IEL) $\mathrm{T}$, and mucosal-associated invariant $\mathrm{T}$ (MAIT) cells, based on known markers [23] (Fig. 2c, d). Some $\mathrm{T}_{\mathrm{N} / \mathrm{CM}}$ cells were further assigned as $\mathrm{T}_{\mathrm{N}}$ and $\mathrm{T}_{\mathrm{CM}}$ clusters based on their gene signatures (Fig. 2c, d, Additional file 1: Figure S13A, B). Both $\mathrm{CD}^{+}$and $\mathrm{CD}^{+} \mathrm{T}$ cell clusters showed a distribution pattern in an organspecific manner (Additional file 1: Figure S13C, D), and each of them had differentially expressed genes (Additional file 1: Figure S13A, B and Additional file 4: Table S22, S23). An overlapping of clusters was also observed between organs (such as blood and marrow), suggesting the sharing of common $\mathrm{T}$ cell subtypes (Additional file 1: Figure S13D).

To better understand the developmental state of $\mathrm{T}$ cells, we performed trajectory analyses of $\mathrm{CD}^{+}{ }^{+}$and $\mathrm{CD} 8^{+} \mathrm{T}$ cells. We observed that the trajectory trees rooted from $T_{N}$ cells, sprouting into $T_{C M}, T_{h 1}$, and $T_{R M}$ branches for CD4 $4^{+} T$ cells and $T_{N / C M}, T_{E F F}$, IEL, and $\mathrm{T}_{\mathrm{RM}}$ branches for $\mathrm{CD} 8^{+} \mathrm{T}$ cells (Fig. 2e, Additional file 1: Figure S13E, F). $\mathrm{T}_{\mathrm{RM}}$ cells and IEL cells with higher pseudo-time scores were found in $\mathrm{CD} 4^{+}$and $\mathrm{CD} 8^{+}$ $\mathrm{T}$ cells, respectively, suggesting their terminal developmental state (Fig. 2e, Additional file 1: Figure S13E, F). Moreover, there are several $\mathrm{T}_{\mathrm{RM}}$ clusters at the end of other branches with mediated scores for both $\mathrm{CD} 4^{+}$and $\mathrm{CD} 8^{+} \mathrm{T}$ cells, indicating the middle developmental state of these clusters. The $T_{R M}$ clusters with different developmental states showed an organ-specific pattern (Additional file 1: Figure S13E, F), while their origin from the marrow or spleen was unclear due to the limited number of cells. These observations reveal the heterogeneity in the developmental states of both $\mathrm{CD} 4^{+}$ and $\mathrm{CD} 8^{+}$cells in human organs.

Transcription factors (TFs) have been demonstrated as important regulators of gene expression and with ability to shape different phenotypes of $\mathrm{T}$ cells [24]. We therefore performed single-cell regulatory network inference and clustering (SCENIC) analysis to assess TFs underlying differential gene expression in $\mathrm{T}$ cells. We identified well-defined and cell subtype-specific TFs for $\mathrm{CD}^{+}$(Fig. 2f) and $\mathrm{CD}^{+} \mathrm{T}$ cell (Fig. 2g) clusters (Additional file 4: Table S24, S25), such as higher activity of FOXP3 [24] and BATF [25] in $\mathrm{T}_{\text {reg }}$ cells, and upregulation of $L E F 1, M Y C, T C F 7$ [26], and KLF2 [27] in $\mathrm{T}_{\mathrm{N}}$ cells and TBX21, STAT1, and IRF1 in $\mathrm{T}_{\mathrm{EFF}}$ cells [24, 28, 29] (Fig. 2f, g and Additional file 1: Figure S13G, H). In addition, many other poorly investigated TFs were also observed in both $\mathrm{CD}^{+}$and $\mathrm{CD}^{+} \mathrm{T}_{\mathrm{RM}}$ cell clusters, such as the upregulation of several AP-1 dimerization partners (FOS, JUN, JUND, FOSL2, and ATF3), REL, and RELB (Fig. 2f, g and Additional file 1: Figure S13G, H). Collectively, these results indicate that the combinations of multiple TFs regulate $\mathrm{T}$ cell development to maintain the heterogeneous states of $\mathrm{CD} 4^{+}$and $\mathrm{CD} 8^{+} \mathrm{T}$ cells.

To better investigate the clonalities and dynamic relationships among $\mathrm{T}$ cell subtypes across tissues, we performed TCR clonal typing accompanied with transcriptome analysis (Fig. 1a). After stringent QC filters, we identified 5183 TCR clonotypes with unique heterodimer $\alpha$ and $\beta$ chains among 8394 T cells (45.89\% of the whole 18,292 $\mathrm{CD}^{+}$and $\mathrm{CD} 8^{+} \mathrm{T}$ cells), including $3248 \mathrm{CD}^{+} \mathrm{T}$ cells and $5146 \mathrm{CD}^{+} \mathrm{T}$ cells. Among 


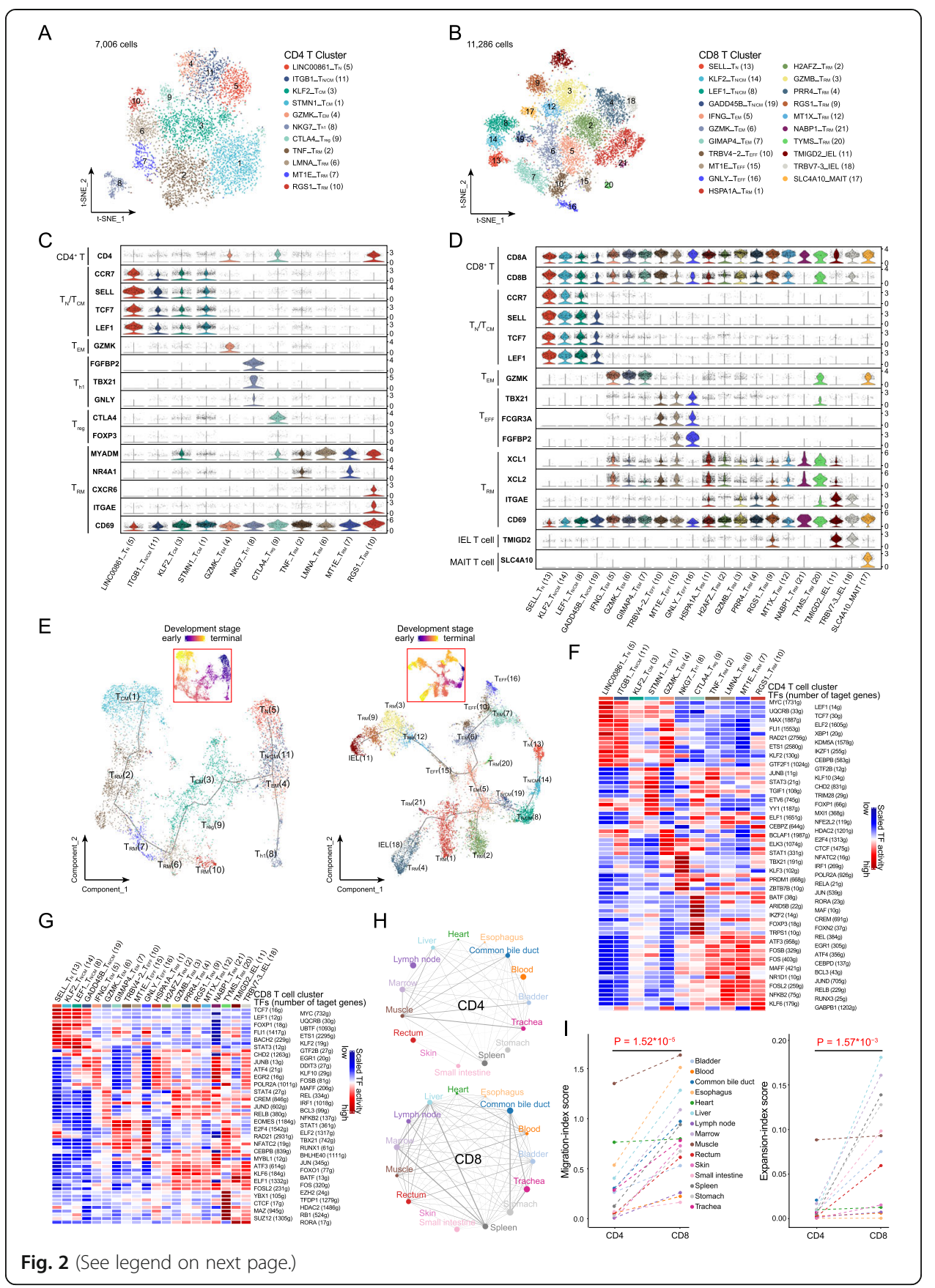


(See figure on previous page.)

Fig. 2 The heterogeneity, development, and clonality of T cells in human organs. a, b t-SNE plots of 7006 $\mathrm{CD}^{+}$(a, 11 clusters) and 11,256 CD8 ${ }^{+}$(b, 21 clusters) T cells from 15 organ tissues. Each dot represents one cell. Each color-coded region represents one cell cluster, which is indicated on the right. $\mathbf{c}$, $\mathbf{d}$ Violin plots showing the normalized expression of marker genes for each $\mathrm{CD}^{+}(\mathbf{c})$ and $\mathrm{CD}^{+}(\mathbf{d}) \mathrm{T}$ cell cluster as indicated at the bottom. For each panel, the $y$-axis shows the normalized expression level for a marker gene as indicated on the left. Marker genes were also grouped according to functional cell types. $\mathbf{e}$ Pseudo-time trajectory analysis of all $\mathrm{CD}^{+}$(left panel) and $\mathrm{CD} 8^{+} \mathrm{T}$ cells (right panel) with high variable genes. Each dot represents one cell and is colored according to their cluster above: $\mathbf{a}$ for $\mathrm{CD}^{+}$and $\mathbf{b}$ for $\mathrm{CD}^{+}$. The inset t-SNE plot shows each cell with a pseudo-time score from dark blue to yellow, indicating early and terminal states, respectively. $\mathbf{f}, \mathbf{g}$ Heat maps of the activation scores of each $\mathrm{T}$ cell cluster for expression regulated by transcription factors (TFs). T cell clusters are indicated on top, and the scores were estimated using SCENIC analysis. Only shows the top $15 \mathrm{TFs}$ for $\mathrm{CD} 4^{+} \mathrm{T}$ cells (f) and the top 10 for $\mathrm{CD} 8^{+} \mathrm{T}$ cells $(\mathbf{g})$, with the highest difference in expression regulation estimates between each cluster and all other cells, under a Wilcoxon rank-sum test. $\mathbf{h}$ Sharing intensity of TCR clones in $\mathrm{CD}^{+}$(top panel) and CD8 ${ }^{+}$ (bottom panel) T cells between different organ samples. Each line represents a sharing of TCR between two organs at the ends, and the thickness of the line represents a migration-index score between paired organs calculated by STARTRAC. The sizes of the dots are shown according to the logarithm to the base 2 of the size of T cell clones in organs with different colors. i Migration- (left panel) and expansion-index (right panel) scores of $\mathrm{CD}^{+}$and $\mathrm{CD} 8^{+} \mathrm{T}$ cells of each tissue calculated and compared using STARTRAC with a paired Student's $t$ test

them, 4645 cells (2906 CD4 ${ }^{+}$and $1739 \mathrm{CD}^{+} \mathrm{T}$ cells) had a unique TCR clonotype for each, while the remaining 3749 cells $\left(342 \mathrm{CD}^{+}\right.$and $3407 \mathrm{CD}^{+} \mathrm{T}$ cells) shared two or more of the 538 TCR clonotypes (Additional file 4: Table S26, S27). We observed similar numbers of $\mathrm{V}$ and $\mathrm{J}$ segments for the TCR $\alpha$ chain in both $\mathrm{CD} 4^{+}$and $\mathrm{CD} 8^{+} \mathrm{T}$ cells, both of which shared $60 \%$ and $40 \%$ of the top 10 frequent $\mathrm{V}$ and $\mathrm{J}$ segments, respectively (Additional file 1: Figure S14A, B). By contrast, the diversity of the V segment was much higher than that of the J segment for $\beta$ chain in both $C D 4^{+}$and $C D 8^{+} \mathrm{T}$ cells, which shared $40 \%$ and $80 \%$ of the top 10 frequent $\mathrm{V}$ and $\mathrm{J}$ segments, respectively (Additional file 1: Figure S14A, B). Although more $\mathrm{CD}^{+} \mathrm{T}$ cells were detected than $\mathrm{CD} 4^{+} \mathrm{T}$ cells among all tissues, no significant difference in clone sizes (the number of unique clonotypes) was observed between the two cell populations (Additional file 1: Figure S14C). Singular cells of unique TCR clonotypes were prevalent for $\mathrm{CD}^{+} \mathrm{T}$ cells among tissues, except that a higher proportion of multiple cells with identical TCR clonotypes or clonal expansions were observed in the muscle, common bile duct, and marrow (Additional file 1: Figure S14D top panel). Clonal expansions were much commonly found for $\mathrm{CD}^{+} \mathrm{T}$ cells in all tissues (Additional file 1: Figure S14D bottom panel).

To investigate the clonotype distribution of $\mathrm{T}$ cells across tissues, we evaluated the ability of sharing TCR for each tissue with others. We observed a more intensive and broader sharing of TCR clonotype for $\mathrm{CD}^{+}$than $\mathrm{CD} 4^{+} \mathrm{T}$ cells across tissues (Fig. 2h). A higher migration capacity, reflected in the migration-index score of tissues, was found in $\mathrm{CD}^{+} \mathrm{T}$ cells than in $\mathrm{CD} 4^{+} \mathrm{T}$ cells (Fig. 2i left panel). Moreover, higher expansion ability but lower diversity was observed in $\mathrm{CD}^{+} \mathrm{T}$ cells in each tissue compared with $\mathrm{CD}^{+} \mathrm{T}$ cells (Fig. 2i right panel, Additional file 1: Figure S14E). Cells with clonal expansion had considerable proportions in $\mathrm{T}_{\mathrm{EM}}, \mathrm{T}_{\mathrm{EFF}}, \mathrm{T}_{\mathrm{RM}}$, and IEL clusters of $\mathrm{CD} 8^{+} \mathrm{T}$ cells (Additional file 1: Figure S14F bottom panel), and $\mathrm{T}_{\mathrm{h} 1}$ and RGS1_ $\mathrm{T}_{\mathrm{RM}}$ of CD4 ${ }^{+} \mathrm{T}$ cells (Additional file 1: Figure S14F top panel). We further evaluated the clonal expansion and transition (clonotype sharing ability of each subpopulation) of each $\mathrm{T}$ cell cluster, which revealed significantly stronger expansion and transition abilities of CD8 ${ }^{+}$ compared with $\mathrm{CD}^{+}{ }^{+} \mathrm{T}$ cells (Additional file 1: Figure S14G, H). This is consistent with 
the stronger sharing links of clonotypes between subtypes of $\mathrm{CD}^{+}$than $\mathrm{CD} 4^{+} \mathrm{T}$ cells (Fig. 2h, Additional file 1: Figure S14I, J). Moreover, we observed the clonal expansion of certain $\mathrm{CD}^{+} \mathrm{T}$ cells $\left(\mathrm{T}_{\mathrm{EFF}}, \mathrm{T}_{\mathrm{RM}}, \mathrm{T}_{\mathrm{EM}}\right.$, and IEL $\mathrm{T}$ cells) distributed across multiple tissues with different developmental states (Additional file 1: Figure S15A), suggesting that most of these cells recognizing the same antigens propagate and migrate more intensively than $\mathrm{T}$ cells of other types. In addition, we observed consistent results of TCR diversity using $\beta$ chains (Additional file 1: Figure S15B-D). These observations based on TCR tracing suggest the widespread of diverse T cells across the human body through clonal expansion and transition.

\section{The heterogeneity of B cells and plasma cells}

Cluster analysis revealed 14 distinct cell clusters among 10,100 B and plasma cells from 11 organ samples, including nine B (CD20) and six plasma cell (SDC1) clusters (Fig. 3a). We observed the predominance of B cells over plasma cells in all tested organs except for the esophagus and rectum (Fig. 3b). Differential gene expression (DEG) analysis revealed that $\mathrm{B}$ cells exhibited distinct gene profiles from plasma cells (Additional file 5: Table S28 and Additional file 1: Figure S16A). Although CD27 is a canonical marker of memory B cells [30], we observed a low transcription level of CD27 in memory B cells, but a higher level in plasma cells (Fig. 3c). TCL1A was significantly expressed in two naïve $B$ cell clusters with distinct gene expression profiles, TCL1A_ly_naive_B from the lymph nodes and TCL1A_naive_B from multiple tissues, compared with the other $\mathrm{B}$ and plasma cell clusters (Fig. 3a, Additional file 1: Figure S16A). Moreover, TCL1A was exclusively expressed in non-CD27-expressing B cell clusters and had a significantly reverse correlation with $C D 27$ transcription (Pearson's $R=-0.84, P=0$; Fig. 3c, Additional file 1: Figure S16B). Given that TCL1A has been reported as an important gene in B cell lymphomas [31], these findings suggest that TCL1A might be a novel marker of naïve $B$ cells.

B cells are professional antigen-presenting cells (APCs) with high expression of CIITA and MHC class II genes, which are silenced during the differentiation to plasma cells [32]. We examined the antigen-presenting ability of B and plasma cell clusters, using the antigen-presenting score (APS) based on the expression of signature genes related to antigen-presenting (see the "Materials and methods" section). Interestingly, plasma cells had a much lower APS for extracellular antigens compared with B cell clusters (Additional file 1: Figure S16C), while they had a much higher APS for intercellular antigens than B cells, suggesting the different abilities to present antigens between the two cell types. We also investigated the potential biological function of different cell clusters using Gene Ontology enrichment (GO) analysis. Potential biological functions of $\mathrm{B}$ cells were found to be enriched in immune response (for example, "responding to activation of immune"), and those of plasma cells were associated with protein synthesis (including "signal peptide processing," "ER to Golgi vesicle-mediated transport," and "protein folding"; Fig. 3d). In addition, gene set enrichment analysis (GSEA) showed that TCL1A_naive_B cells were enriched in "oxidative phosphorylation" and "fatty acid metabolism" pathways (Additional file 1: Figure S16D). These observations suggest distinct biological functions of $\mathrm{B}$ and plasma cells, although plasma cells were derived from B cells. 


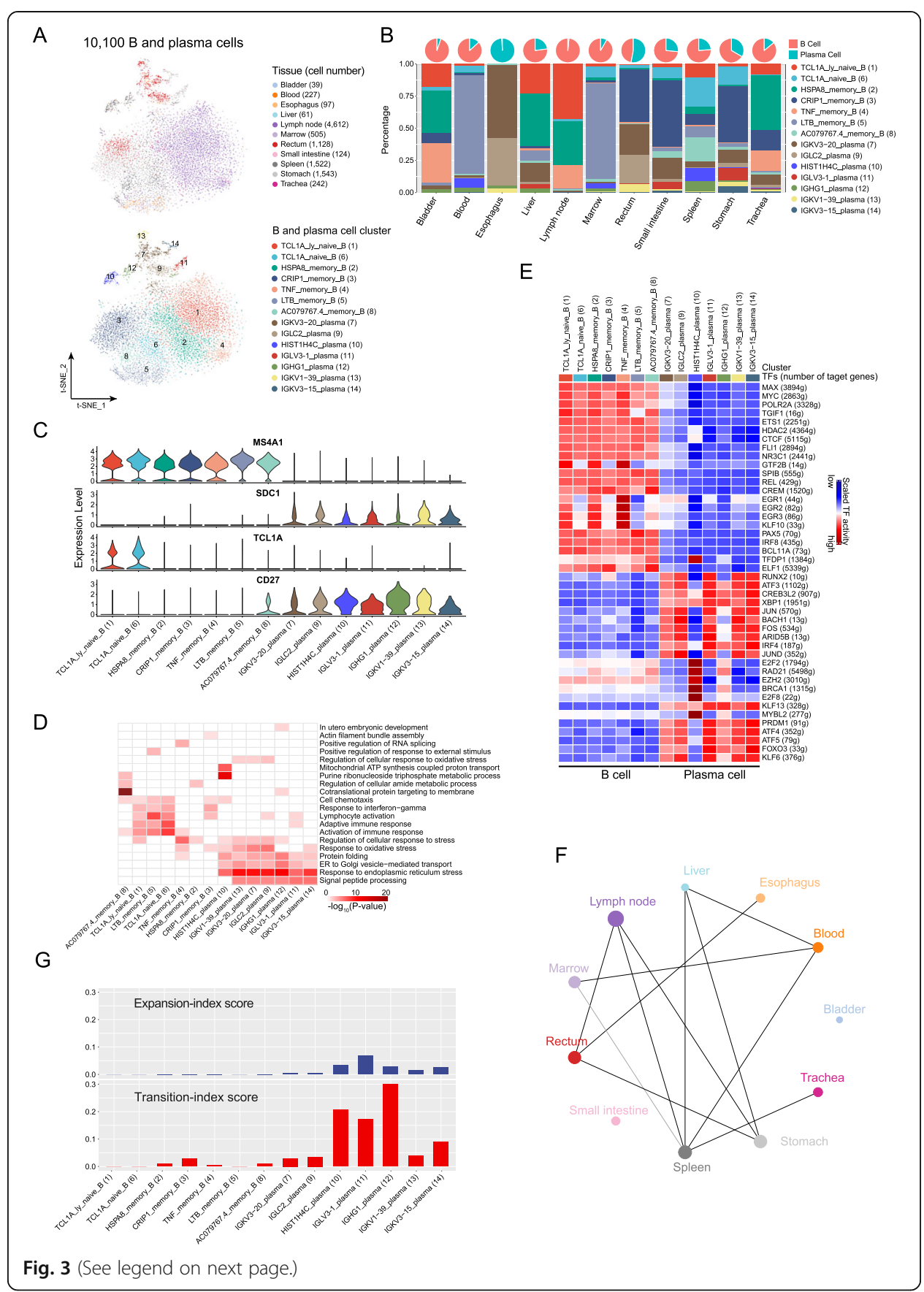


(See figure on previous page.)

Fig. 3 The heterogeneity and clonality of B cells in human organs. a t-SNE plots showing 14 clusters $(10,100$ cells $)$ of B and plasma cells. Each dot represents a cell, colored according to the origin of tissue (top panel) and cell subtype (bottom panel). $\mathbf{b}$ Distribution of B and plasma cells in each organ. Pie charts on top illustrate the proportions of $B$ and plasma cells in each organ. The stacked bars represent the percentage of each cluster in the indicated organ. $\mathbf{c}$ Violin plots of the normalized expression of marker genes for B (MS4A1), plasma cells (SDC1), naïve B cell (TCL1A), and memory B cells (CD27). For each panel, the $y$-axis shows the normalized expression level for a marker gene as indicated on the title, and the $x$-axis indicates cell clusters. $\mathbf{d}$ Gene Ontology enrichment analysis results of B and plasma cell clusters. Cell clusters as indicated at the bottom are colored according to their $-\log _{10} P$ values in columns. Only the top 20 significant $\mathrm{GO}$ terms ( $P$ value $<0.05$ ) are shown in rows. e Heat map of the activation scores of each $\mathrm{B}$ and plasma cell cluster for expression regulated by transcription factors (TFs). Cell clusters are indicated on top, and the scores were estimated using SCENIC analysis. It shows the top 10 TFs with the highest difference in expression regulation estimates between each cluster and all other cells, tested with a Wilcoxon rank-sum test. $\mathbf{f}$ Sharing intensity of BCR clones between different organs. Each line represents a sharing of BCR between two organs at the ends, and the thickness of the line represents a migration-index score between paired organs calculated using STARTRAC. The size of the dot is shown as the logarithm to the base 2 of the size of $B$ and plasma cell clones in each organ. $\mathbf{g}$ Expansion- (top panel) and transitionindex (bottom panel) scores of each B and plasma cell cluster calculated using STARTRAC

TFs play a critical role in the differentiation of B cells to plasma cells, engaging B cells with effector or memory functions [33]. Single-cell regulatory network inference and clustering (SCENIC) analysis revealed that TFs exhibited similar activities in $\mathrm{B}$ and plasma cells but with distinct patterns between the two populations (Fig. 3e, Additional file 5: Table S29). TFs with higher activity were found in B cells, including $M Y C$ [34] and REL [35], which have been known to modulate $\mathrm{B}$ cell development, as well as other TFs that have not been characterized in B cells, such as EGF receptors (EGFR1/2/3) and TGIF1. Likewise, TFs enriched in plasma cells included PRDM1, XBP1, FOS, and IRF4, which play important roles in the development of plasma cells [33]. Moreover, many TFs with unknown roles were found, including ATF3, ATF4, and ATF5. Consistently, we observed higher activity of these TFs in an independent human cell landscape (HCL) dataset published recently [20], including $M Y C$, IRF8, and REL in B cell clusters and XBP1, PRDM1, and CREB3L2 in plasma cell clusters (Additional file 1: Figure S16E, F, and Additional file 4: Table S30, S31). Taken together, these results suggest that various TFs might regulate the development of B cells into plasma cells.

To explore the clonalities of $\mathrm{B}$ and plasma cell clusters across organ tissues, we performed a single-cell BCR sequencing analysis. After stringent QC filters, 6741 out of 10,100 cells were assigned to 6480 clonotypes, among which 6330 clonotypes were presented by singular cells and 150 by multiple cells (Additional file 4: Table S32). We observed various usage of $\mathrm{V}$ and $\mathrm{J}$ gene segments for both heavy and light chains of immunoglobulin genes, with a preferred usage of some particular variable segments (Additional file 1: Figure S16G-I). Unlike T cells, clonal diversity was common for B cells among all the organs, while clonal expansion of B cells was limited and restricted to the spleen, rectum, and stomach (Additional file 1: Figure S16J). Moreover, less sharing of BCR clonotypes between B and plasma cells was observed across organs compared with $\mathrm{T}$ cells (Fig. $2 \mathrm{~h}$ and Fig. 3f). In addition, BCR analysis showed lower expansion and transition abilities of B cells compared with plasma cells and $\mathrm{T}$ cells (Fig. 3g, Additional file 1: Figure S14G, and Additional file 1: Figure S16K, L), which might be due to an insufficient representation of the richness of the diverse B cell repertoire with the limited number of clonal B cells detected. 


\section{The heterogeneity of myeloid cells}

We obtained 5587 myeloid cells from 15 organ tissues, which were grouped into 18 distinct clusters (Fig. 4a, b). Based on the differential expressions of marker genes, we further identified seven monocyte clusters, eight macrophage clusters, and three dendritic cell (DC) clusters (Fig. 4a, b, and Additional file 1: Figure S17A). The hierarchical cluster analysis revealed that all classical monocytes were closely related as a branch node, as were non-classical monocytes and intermediate monocytes (Fig. 4c). All macrophages were grouped together and separated from monocytes, except that one subset of macrophages (C3: SDC3_Mac) was closely related to intermediate and non-classical monocytes (Fig. 4c). DEG analysis showed that each myeloid cell cluster had a specific gene signature, suggesting inter-cell heterogeneity among monocytes, macrophages, and DCs (Fig. 4d, Additional file 6: Table S33). All clusters contained cells from multiple tissues, except for FN1_Intermediate_Mon (C4) and CCL20_Intermediate_Mon (C10) in the rectum and Langerhans clusters (C15: Langerhans) in the skin, suggesting similar transcriptional profiles and origins of these myeloid cells (Fig. 4a, b). Monocytes showed a predominance in 11 of the 15 tested tissues, except for the esophagus, heart, lymph nodes, and skin, where macrophages and DCs accounted for more than $50 \%$ of all myeloid cells (Additional file 1: S17A).

Considering two potential origins of macrophages in multiple tissues from circulating monocytes and embryonic progenitor cells [36-38], we examined the connection between macrophages and monocytes. First, we observed the coexistence of macrophages and monocytes in the same organs (Fig. 4a, b, and Additional file 1: Figure S17A). Second, trajectory analysis revealed that the classical monocytes had initial state at the root and sprouted into branches with more developed states of monocytes and then macrophages either directly or via two intermediate monocytes (Fig. 4e, Additional file 1: Figure S17B, C). Notably, high expression of proliferation marker genes including MKI67 and PCNA were exclusively detected in the HISTIH4C_Mac macrophages $(\mathrm{C} 12)$ in the bladder, esophagus, heart, and rectum (each with more than five of the cells; Additional file 1: Figure S17D). Gene set variation analysis (GSVA) also revealed that most of the macrophage clusters had a higher enrichment score of MYC and E2F target pathways (Additional file 1: Figure S17E). We further performed a trajectory analysis of intestinal monocytes and macrophages derived from our and published datasets [39]. We observed a terminal state for both the embryonic and adult macrophages according to pseudo-time scores (Additional file 1: Figure S17F). Moreover, we observed a clear differentiation trajectory of the macrophages in the adult rectum from the intermediate monocytes (CCL20_Intermediate_Mon) to the tissue macrophages (HIST1H4C_Mac). Interestingly, the embryonic macrophages (Embryo_Mac) were found alongside the differentiation trajectory from the intermediate monocyte (FN1_Intermediate_Mon) to the tissue macrophages (HIST1H4C_Mac), suggesting that the embryonic macrophages may contribute to the tissue macrophages through local expansion. Consistently, a high expression of the proliferation marker gene PCNA was detected in the terminal macrophages (Additional file 1: Figure S17G). Taken together, these observations suggest that circulating monocytes might give rise to macrophages in organ tissues and that the local microenvironment may contribute to the expansion and proliferation of different embryo-derived macrophage populations, especially tissue-resident macrophages, in the bladder, esophagus, heart, and rectum. 


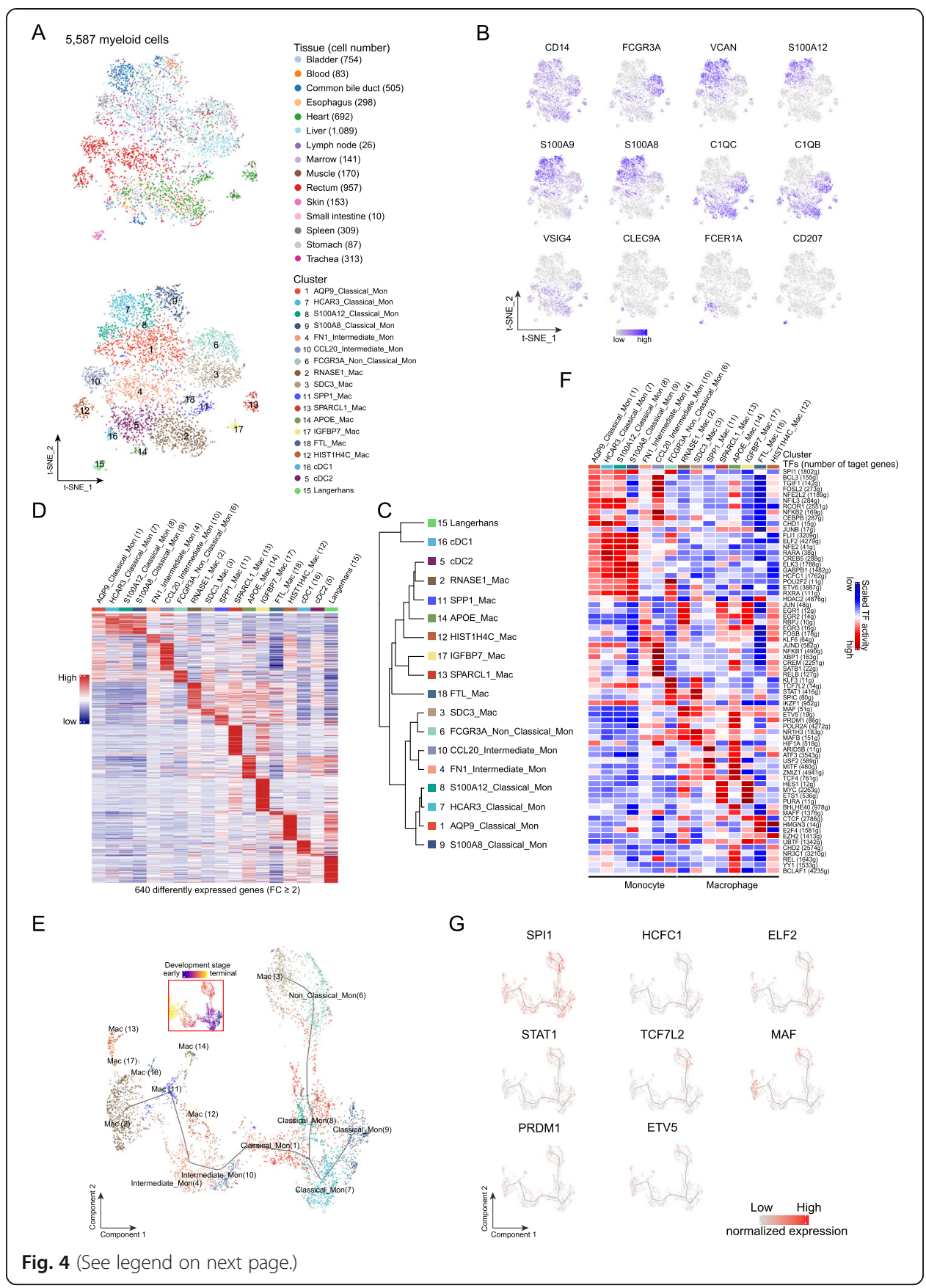


(See figure on previous page.)

Fig. 4 Heterogeneity and developmental stages of myeloid cells. a t-SNE plots of 5587 myeloid cells. Each dot represents one cell, colored according to their tissue origins (top panel) or cell clusters (bottom panel) as indicated on the right. $\mathbf{b} \mathrm{t}-\mathrm{SNE}$ plots of the normalized expression of marker genes for monocytes (S100A8/9/12 and VCAN) and macrophages (pan-marker: C1QC, C1QB, and VSIG4), CDC1 (CLEC9A), CDC2 (FCER1A), and Langerhans (CD207) as well as subpopulation-specific genes (CD14 and FCGR3A). Each dot represents one cell, with a color from gray to blue representing the expression level from low to high. $\mathbf{c}$ Dendrogram of 18 clusters based on their normalized mean expression values (correlation distance metric, complete linkage). Only genes with In(fold change) above 0.25 , p.adjust $<0.05$, and pct. $1 \geq 0.2$ in each cluster were included in the calculations. $\mathbf{d}$ Heat map showing the expression profiles of each myeloid cell cluster as indicated on top. The expression of 640 genes in each cell cluster with $F C \geq 2$ and p.adjust $<0.05$ are shown as lines, colored from blue to red according to the expression from low to high. e Pseudo-time trajectory analysis of monocytes/macrophages with high variable genes. Each dot represents one cell and is colored according to their clustering in $\mathbf{a}$. The inset t-SNE plot shows each cell with a pseudo-time score from dark blue to yellow, indicating early and terminal states, respectively. $\mathbf{f}$ Heat map of the activation scores of each monocyte and macrophage subtype for gene expression regulated by transcription factors (TFs). Cell clusters are indicated on top, and the scores were estimated using SCENIC analysis. Only the top 10 TFs are shown with the highest difference in expression regulation estimates between each cluster and all other cells, tested with a Wilcoxon rank-sum test. $\mathbf{g}$ Plots showing the normalized expression of representative TFs in $\mathbf{f}$ along the pseudo-time trajectory maps corresponding to $\mathbf{e}$. Each dot in one plot shows the expression of the indicated gene in the plot, colored from gray to red, indicating low and high expression, respectively. SPIP1, HCFC1, and ELF2 for classical monocytes; enhanced expression of STAT1 and TCF7L2 for non-classical monocytes and SDC3_Mac (3); MAF, PRDM1, and EVT5 for most

non-classical monocytes

TFs such as SPI1 are involved in the development of monocytes to macrophages [40, 41]. However, how TFs regulate their development in the normal human body is still unclear. TF activity analysis revealed a similar activation of certain TFs among the four classical monocytes, which is consistent with their close similarity in gene signatures (Fig. 4d, $\mathrm{f}$ and Additional file 6: Table S34). A high activation of SPI1 was determined in classical monocytes, non-classical monocytes, and SDC3_Mac cells, suggesting its involvement in the development of these cells. We also identified several TFs with high activity in the four classical monocyte clusters, including HCFC1, ELF2, ETV6, ELK3, and NFE2 (Fig. 4f, g). Their roles in maintaining the classical state of monocytes have yet to be investigated. Non-classical monocytes and macrophages shared several activated TFs, such as TCF7L2, STAT1, KLF3, NR1H3, and SPIC, which is consistent with their close pattern in the hierarchical cluster analysis (Fig. 4c, f, g). We observed that multiple poorly characterized TFs were highly activated in the intermediate, non-classical monocytes and macrophages, other than classical monocytes, including POLR2A, MAF, MAFB, PRDM1, ETV5, and ATF3 (Fig. 4c, f, g). We also observed cluster-specific TFs for myeloid cell clusters (Fig. 4f, Additional file 6: Table S34). For instance, the activation of BHLHE4O and NR3C1 was higher in APOE_Mac cells (C14). These results suggest that these unique combinations of TFs help shape the different states of myeloid cells in the normal human body.

It has been demonstrated that myeloid cells could act as professional APCs, with the strongest antigen-presenting ability for DCs [42]. We observed various antigenpresenting abilities for extracellular antigens as reflected by APS for different myeloid clusters (Additional file 1: Figure S17H). Langerhans cells had a stronger antigenpresenting ability than other macrophages and monocytes $\left(P<2.2 \times 10^{-16}\right.$; Additional file 1: Figure $\mathrm{S} 17 \mathrm{H})$. On average, the classical monocytes had the lowest APS among myeloid cell clusters $\left(P<2.2 \times 10^{-16}\right)$. Interestingly, different myeloid cell clusters had a similar APS for presenting intracellular antigen (Additional file 1: Figure S17H). These observations support the varied roles of myeloid cells in antigen presentation. 
The similarity and heterogeneity of epithelial cells from intra- and inter-tissues

We obtained a total of 17,436 epithelial cells from nine tested organ tissues (Fig. 5a). DEG analysis revealed a clear and distinct pattern of gene expression among the tissue samples (Additional file 1: Figure S18A and Additional file 7: Table S35). There were 190 genes with tissue-specific expression ( $\mathrm{FC} \geq 5$, pct. $1 \geq 0.2$; Additional file 7: Table S35 and Additional file 1: Figure S18A), indicating the heterogeneity of epithelial cells among organ tissues, which was further confirmed in the HCL dataset (Additional file 1: Figure S18B). GO analysis also revealed the various biological functions of epithelial cells from different tissues, among which "epithelial cell differentiation" and "regulation of myeloid leukocyte activation" were common pathways enriched in the majority of epithelial cells. This suggests that the cells share common functions in the development of epithelial cells and the regulation of immune response (Additional file 1: Figure S18C).

The epithelial cells were further grouped into 34 clusters (Fig. 5a). Grouping of close clusters was seen in all organ tissues, except for tiny clusters of C25 (rectum and small intestine), C31 (esophagus and skin), and C32 (skin and small intestine), suggesting the heterogeneity of epithelial cells at both the intra- and inter-organ contexts (Additional file 7: Table S36). The 34 clusters showed different expression profiles with 350 signature genes $(F C \geq 5$, pct. $1 \geq 0.2$, and pct. $2 \leq 0.2$ ), most of which were expressed exclusively in one cluster (Fig. 5b, Additional file 1: Figure S18D, F left panel, and Additional file 7: Table S37). Similar results were observed in the previous HCL dataset (Additional file 1: Figure S18B, E, and F right panel, and Additional file 7: Table S38-S40). Hierarchical cluster analysis revealed closer grouping of cell clusters within tissues than across tissues in both our and the HCL datasets (Fig. 5c, Additional file 1: Figure S18E, and Additional file 7: Table S39). Cells from digestive organs including the small intestine, stomach, rectum, and common bile duct were clustered closely, as were the cells from non-digestive tissues including the skin, trachea, and bladder (Fig. 5c, Additional file 1: Figure S19A). Although the esophagus is a digestive organ, the epithelial cells were clustered much closer to the skin cells than the digestive organs' cells. Similarly, the stomach was grouped more closely to non-digestive organs in the HCL dataset, although it is classified as one of the digestive organs together with the ascending colon, colon, sigmoid colon, transverse colon, small intestine, liver, gallbladder, pancreas, and rectum. This might be explained by the different anatomical positions of the specimens (Additional file 1: Figure S19B). A volcano plot showed significantly DEG between the two groups, including 514 upregulated genes with a fold change greater than two (298 in the digestionrelated clusters and 216 genes in the non-digestion-related clusters; Fig. 5d).

To explore the potential functions of cells within each cluster, we performed GO analysis for two groups of cells from the 14 digestion-related clusters and the 20 nondigestion-related clusters, separately. For cells from the digestive system, biological functions related to metabolic process, energy synthesis, and digestion pathways were commonly observed (Fig. 5e). For the non-digestion-related cells, the "regulation of endopeptidase activity" and "epidermis development" were the strongly enriched pathways (Fig. 5f). Interestingly, GSVA in both our AHCA and the HCL datasets revealed significantly altered pathways between the digestive and non-digestive clusters, including multiple metabolic pathways with elevated activity in the digestive clusters, such as "Fatty acid metabolism," "Citric acid cycle," "Protein modification," and "Pyrimidine 


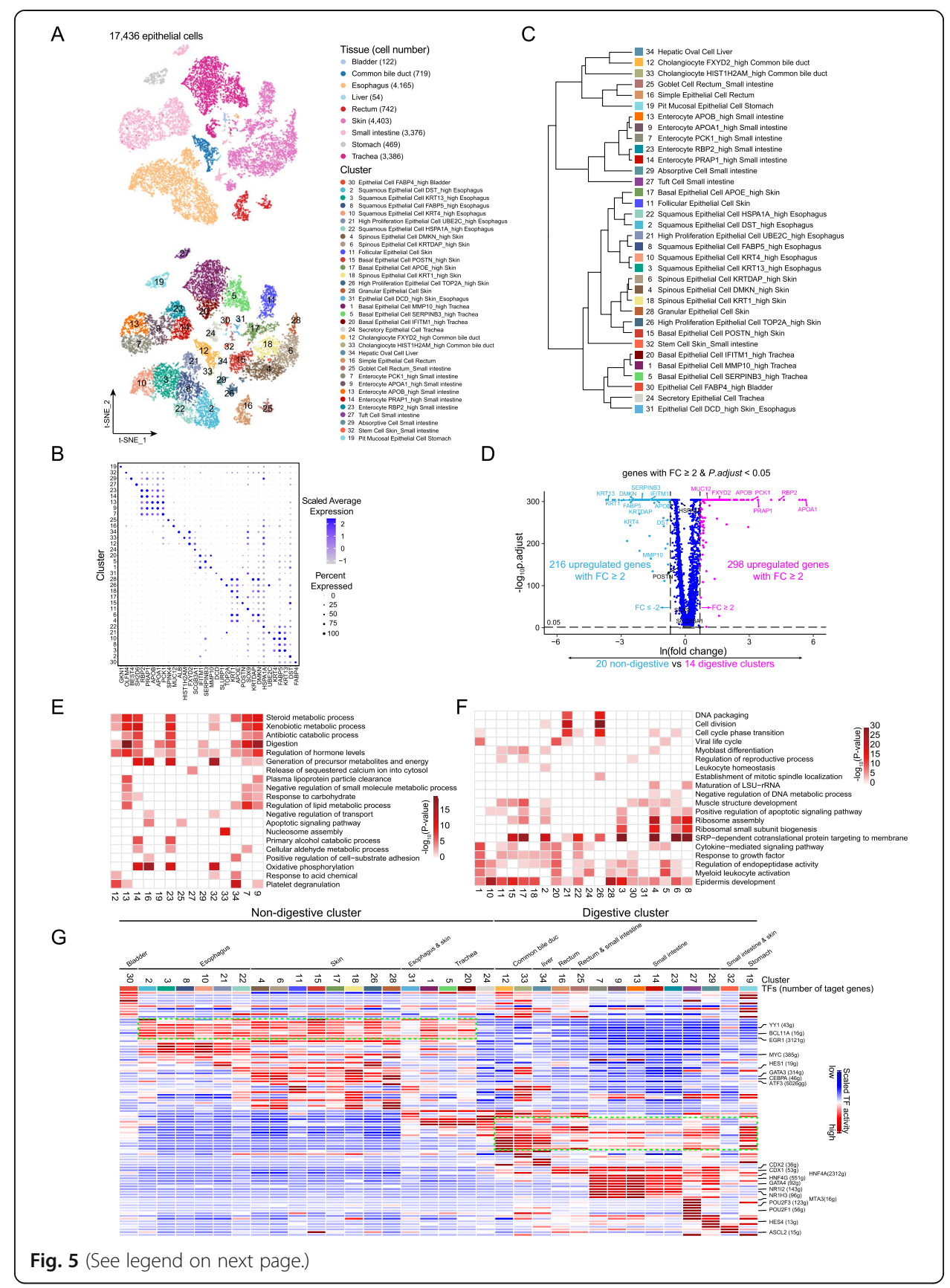


(See figure on previous page.)

Fig. 5 The heterogeneity of epithelial cells inter- and intra-organ tissues. a t-SNE plots of 17,436 epithelial cells. Each dot represents one cell, colored according to their origins of tissues (top panel) or cell clusters (bottom panel). b Dot plot visualizing the normalized expression of marker genes for each epithelial cluster. Cell cluster at $y$-axis was coded in numbers on the left, corresponding to that in $\mathbf{a}$. Marker genes are shown at the $x$-axis. The size of the dot represents the percentage of cells with a cell type, and the color represents the average expression level. c Dendrogram of 34 clusters based on their normalized mean expression values (correlation distance metric, complete linkage). Only genes with fold change above 1.5, p.adjust $<0.05$, and pct. $1 \geq 0.2$ in each cluster were included in the analysis. $\mathbf{d}$ Volcano plot shows the DEGs between the 14 digestive and 20 non-digestive related clusters. Labeled genes are markers for each cluster in $\mathbf{b}$. e, $\mathbf{f}$ Gene Ontology enrichment analysis results of each epithelial cell cluster in the digestive organs (e) and non-digestive organs (f). Cell clusters in columns are coded as numbers at the bottom, correspond to that in $\mathbf{a}$, and are colored according to their $-\log _{10} P$ values, with white to red for low to high enrichment of a GO term in a row indicated on the right. Only the top 20 significant $G O$ terms $(P$ value $<0.05)$ are shown. $\mathbf{g}$ Heat map of the activation scores of epithelial cell subtypes for gene expression regulated by transcription factors (TFs). Cell clusters are indicated on top, and the scores were estimated using SCENIC analysis. Only the top 10 TFs are shown with the highest difference in expression regulation estimates between each cluster and all other cells, tested with a Wilcoxon rank-sum test. The cluster numbers are in reference to those in $\mathbf{a}$. Cell clusters are grouped according to the origin of organ and their digestive or non-digestive function as indicated on top

metabolism," as well as enrichment of "Epithelial mesenchymal transition" and "TNFA signal via NFKB" in the non-digestive epithelial cells, suggesting the enhanced metabolic activity of digestive epithelial cells (Additional file 1: Figure S19C, D). As we observed the enrichment of immune-related pathways in most of the cell clusters (Fig. 5e, f), we examined their antigen-presenting abilities, which revealed a weaker antigenpresenting ability for epithelial cells from the skin and esophagus to present both intraand extracellular antigens in our AHCA dataset but a higher antigen-presenting ability for epithelial cells from the lungs in the HCL dataset (Additional file 1: Figure S20A).

Next, we investigated the contribution of TFs in regulating the heterogeneous transcriptional profiles of epithelial cells in and between organs. SCENIC analysis revealed that the regulation of TFs was similar among cell clusters within a same tissue but was very different among cell clusters between tissues (Fig. 5g, Additional file 7: Table S41). Interestingly, the digestion-associated epithelial clusters exhibited similar activation of TFs, as did the cell clusters belonging to the non-digestive tissues including the trachea, skin, and esophagus (Fig. 5g, Additional file 7: Table S41). We also observed some cluster-specific TFs in the skin (CEBPA, HES1, GATA3, and ATF3), small intestine epithelial cells (HNF4G, NR1H3, NR1I2, HNF4A, CDX1, and CDX2), tuff cells (MTA3, POU2F1, and POU2F3), absorptive cells (HES4), and stem cells (ASCL2; Fig. 5g). Moreover, we identified 204 TFs in our AHCA dataset, which overlapped with about 60\% (201) of all clusterspecific TFs in the HCL dataset. A similar activation pattern of TFs was observed between our AHCA and the HCL datasets for digestive-associated tissues (except for the pancreas) and non-digestive tissues (except for the lungs; Additional file 1: Figure S20B and Additional file 7: Table S42). Together, these observations suggest that TFs may contribute to the heterogeneity of epithelial cells across tissues and epithelial cells from tissues with similar functions may share a similar activation pattern of TFs.

\section{The similarity and heterogeneity of stromal cells}

Endothelial cells (ECs) line up in a monolayer and form the interior surface of blood and lymphatic vessels as well as heart chambers. We identified a total of 6932 ECs, 
including 6681 blood endothelial cells (BECs, marked with VWF) and 251 lymphatic endothelial cells (LECs, marked with LYVE1). BECs and LECs could be further grouped into 11 and two clusters, respectively, with unique gene signatures (Additional file 1: Figure S21 A-C and Additional file 8: Table S43). Although various cell clusters were identified in a tissue, hierarchical cluster analysis revealed that the cell clusters from the same tissue were grouped closer than those between two different tissues (Additional file 1: Figure S21D), such as FABP4_BEC and TNFRSF4_BEC, and APOC1_BEC from the muscle. Interestingly, a group of LECs (FCN3_LEC) were strictly identified in the liver and expressed only two of the four genes for LECs (PECAM1 and LYVE1; but not PDPN and PROX1 [43]; Additional file 1: Figure S21B, E) and other liver-specific markers $(C D 4, C D 14, F C N 2 / 3, O I T 3$, and $C L E C 4 G)$. The other group of LECs (CCL21_LEC) were from tissues except for the liver, with exclusively high expression of CCL21, the protein which binds the chemokine receptor 7 (CCR7) and promotes adhesion and migration of various immune cells [44]. This suggests that these LECs have a higher potential to attract immune cells than the LECs in the liver. GO analysis results revealed that BECs and LECs from most of the clusters had common endothelial functions including "blood vessel development" and "response to wounding," as well as functions regulating immune response (Additional file 1: Figure S21F). Moreover, the cells from each cluster were shown to have specific biological functions, including "endocrine processing" for liver BECs (TIMP1_BEC), "cellular extravasation" and "adaptive immune response" for heart BECs (ACKR1_BEC), and "macrophage migration" for non-liver LECs (CCL21_LEC). We further examined the APS for each cell cluster, which revealed a higher ability in presenting extracellular antigens for BECs from the skin (CTSC_BEC, $P<2.2 \times 10^{-16}$ ) and the LECs in the liver (FCN3_LEC; $P=$ $7.232 \times 10^{-15}$ ) than the other tissues (Additional file 1: Figure S21G).

We also identified a total of 17,690 fibroblasts and smooth muscle cells from nine tissues. These cells were further grouped into 14 fibroblast clusters (11,697 cells, MMP2), four smooth muscle cell clusters (3165 cells, ACTA2), and another five novel clusters assigned as FibSmo (2828 cells; marked with MMP2 and ACTA2; Additional file 1: Figure S22A, B). We observed organ-specific distribution for fibroblasts of different clusters, but a mixture of multiple organs for smooth muscle cell clusters (Additional file 1: Figure S22A), which is consistent with previous findings in a mouse model [9]. DEG analysis revealed that cells from each cluster had a unique signature (Additional file 1: Figure S22C and Additional file 8: Table S44). Hierarchical cluster analysis showed that cells from clusters were grouped in an organ-specific manner, namely close distance for those within a same tissue (Additional file 1: Figure S22D). The presence of the novel FibSmo cells was further confirmed by double immunostaining of fibroblast marker MMP2 and smooth muscle cell marker ACTA2 in multiple tissues (Additional file 1: Figure S10). Fibroblasts, smooth muscle cells, and FibSmo cells had distinct gene signatures (Additional file 1: Figure S23A and Additional file 8: Table S45). GO analysis revealed that the three cell types shared classical functions, including "response to wounding" and "tissue remodeling" (Additional file 1: Figure S23B). However, each of them has unique functions. Fibroblasts have specific enrichment of genes that are involved in the "extracellular structure organization," which is consistent with their strong expression of extracellular matrix protein genes (DCN and FBLN2) and genes related to matrix assembly (MFAP5 and SFRP2) and matrix remodeling (MMP2; 
Additional file 1: Figure S23B, C). Smooth muscle cells have specific enrichment of genes related to muscle system processing, including MYH11, MYLK, CAV1, and MEF2C (Additional file 1: Figure S23B, C). By contrast, FibSmo cells exhibited a high and specific expression of PLAT (Additional file 1: Figure S7) and ID1, which were related to clotting and angiogenesis $[45,46]$, as well as a higher expression of COL3A1 and COL1A1 (Additional file 1: Figure S23B, C), which have been involved in wound healing [47], compared with other stromal cells. Moreover, the enrichment of BMP signal genes BMP4 and BMP5 was also observed in the FibSmo cells (Additional file 1: Figure S23B, C and Figure S7). Furthermore, GO analysis revealed that FibSmo cells had enhanced biological functions in "response to wounding" and "growth factor," which is consistent with the functions of their highly expressed signature genes detailed above (Additional file 1: Figure S23B, C).

\section{Complex and broad intercellular communication networks within and between tissues}

Since we observed heterogeneity for cells in each organ tissue, we explored their potential intercellular communication network. CellphoneDB interaction analysis was conducted to explore cell-cell crosstalk of different cell types in various organs based on the repository of ligands, receptors, and their interactions, which mediates cell-cell communication critical to coordinating diverse biological processes [48]. We observed a total of 20,630 significant interactions based on 475 ligand-receptor pairs among cell types and tissues, which varied from 131 in the lymph nodes to 3229 in the skin (Additional file 1: Figure S24A). Among them, MIF_CD74, HBEGF_CD44, MIF_TNFRSF14, CD55_ADGRE5, and APP_CD74 were the top five frequent interacting pairs detected across different cell types (Additional file 1: Figure S24B and Additional file 9: Table S46), suggesting their important roles in mediating crosstalk between different cell types. Next, we focused on the interactions between pairs of the major cell types (Additional file 1: Figure S24C and Additional file 9: Table S47). Myeloid cells were the most active cell type interacting with the other types of cells (6949 inter-cell interactions), especially with epithelial cells ( $24.3 \%$ of the total inter-cell interactions) in the skin and trachea (51.8\%). Interestingly, common interactions were observed between myeloid cells and epithelial cells, with the most frequent ligand-receptor pair HBEGF_CD44 (Fig. 6a, Additional file 1: Figure S25), of which dysregulations were involved in tumor and metastasis initiation [49]. $\mathrm{CD}^{+} \mathrm{T}$ cells were another cell type with intensive interactions with other types of cells (total inter-cell 4290 interactions), especially with myeloid cells (29.9\% of the total inter-cell interactions). The interactions were found mainly in the liver, trachea, and common bile duct (44.6\%). The frequent interaction pairs between $\mathrm{CD}^{+} \mathrm{T}$ and myeloid cells were RPS19_C5AR1, CD55_ADGRE5, MIF_CD74, HBEGF CD44, CD99_PILRA, and ANXA1_FPR1, most of which play important roles in immune regulation [50-52] (Fig. 6b, Additional file 1: Figure S26). CD8 ${ }^{+} \mathrm{T}$ cells also had broad interactions with non-immune cells (Additional file 9: Table S47). For instance, the most frequent interacting chemokine and receptor pair CXCL12_CXCR4 [53] was observed between stromal (fibroblasts, FibSmo, and smooth muscle cells) and $\mathrm{CD} 8^{+} \mathrm{T}$ cells, suggesting the chemoattraction potential of those stromal cells for T cells' migration into tissues (Fig. 6c, Additional file 1: Figure S27). We observed broad interactions with different densities among various organ tissues (Fig. 6d, Additional file 10: Table S48). We noted 


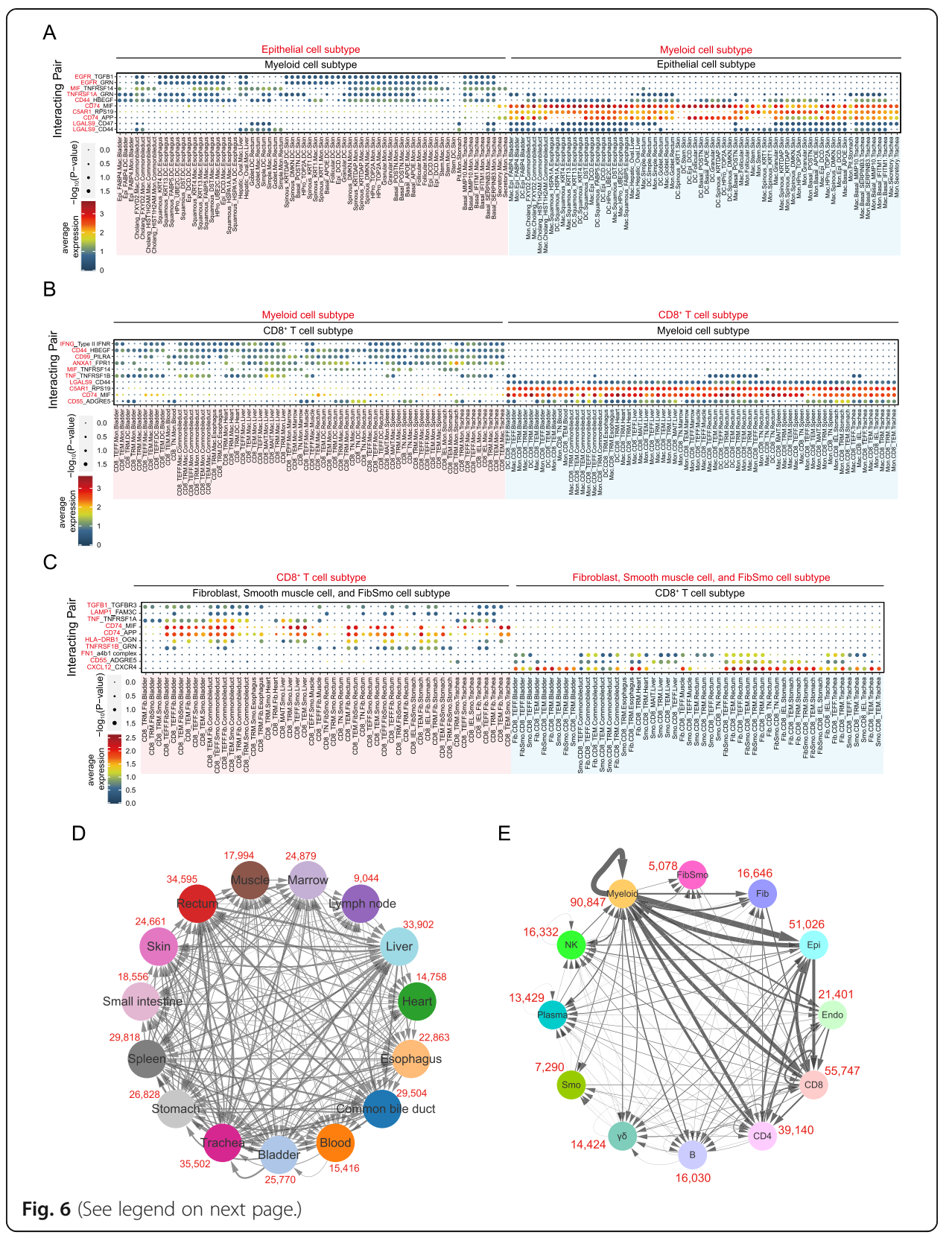


(See figure on previous page.)

Fig. $\mathbf{6}$ Intercellular communication networks among tissues. a-c The top 10 significant ligand-receptor interactions between cells among different organs for epithelial and myeloid cell subtypes (a), myeloid and $\mathrm{CD}^{+} \mathrm{T}$ cell subtypes $(\mathbf{b})$, and $\mathrm{CD} 8^{+} \mathrm{T}$ and stromal cell subtypes (c; fibroblast, smooth muscle cell, and FibSmo cell). An interaction is indicated as color-filled circle at the cross of interacting cell types in a tissue ( $x$-axis) and a ligand-receptor pair ( $y$-axis), with circle size representing the significance of $-\log _{10} P$ values in a permutation test and colors representing the means of the average expression level of the interacting pair. The naming system is as follows: taking an example of "EGFR_TGFB1" in "cholang_FXYD2.Mac.Commonbileduct," the ligand-receptor pair is EGFR (red) and TGFB1 (black), and the circle is colored based on the expression levels of EGFR in cholang_FXYD2 cluster and TFGB1 in Mac cluster in the tissue Commonbileduct. Mon, monocyte; Mac, macrophage; DC, dendritic cell; TRM, tissue-resident memory T cell; TEFF, effector T cell; TGD, $ү \delta$ T cell; MAIT, mucosal-associated invariant T cell; TEM, effector memory T cell; TIEL, intraepithelial T lymphocyte; TN, naïve T cell; Fib, fibroblast; Smo, smooth muscle cell; FibSmo, novel cell type named FibSmo cell; Commonbileduct, common bile duct; Lymphnode, lymph node; Smallintestine, small intestine. For epithelial cells, the full names of each cluster refer to Table S47. $\mathbf{d}$ Connection graph showing the intensity of interactions between one organ to another in colored circles. Interactions were evaluated between major cell types including $\mathrm{CD}^{+} \mathrm{T}$ cell, CD8 ${ }^{+}$T cell, $ү \delta$ T cell, B cell, plasma cell, myeloid cell, NK cell, epithelial cell, fibroblast, smooth muscle cell, FibSmo cell, and endothelial cell. Numbers in red show the total counts of ligand-receptor pairs between the indicated organ and all others, which include only the unique significant interacting pairs between them (average expression $>0$ and $P$ value $<0.05$ ). e Connection graph showing the intensity of interactions within a major cell type or between two major cell types in colored circles. Numbers in red show the total counts of ligand-receptor pairs within or between cell types, which only included the unique significant interacting pairs between them (average expression $>0$ and $P$ value $<0.05)$. CD4, CD4 ${ }^{+} T$ cell; CD8, CD8 ${ }^{+} T$ cell; $\gamma \delta, \gamma \delta T$ cell; B, B cell; Plasma, plasma cell; Myeloid, myeloid cell; NK, NK cell; Epi, epithelial cell; Fib, fibroblast; Smo, smooth muscle cell; FibSmo, FibSmo cell; Endo, endothelial cell

that cells in the trachea had a high density of interaction pairs with multiple tissues including the rectum, liver, and spleen, suggesting the potential regulatory communications between these organs. Interestingly, myeloid, $\mathrm{CD}^{+} \mathrm{T}$, and epithelial cells were the core nodes of cell-cell interactions, which had the greatest number of interacting pairs and enhanced pairwise communications (Fig. 6e, Additional file 10: Table S48).

\section{Discussion}

Here, to the best of our knowledge, we for the first time generated an adult human cell atlas (AHCA), by profiling the single-cell transcriptome for 84,363 cells from 15 organs of one adult donor. The AHCA included 252 cell subtypes, each of which was distinguished by multiple marker genes and transcriptional profiles and collectively contributed to the heterogeneity of major human organs. The AHCA empowered us to explore the developmental trajectories of major cell types and identify regulators and interacting networks in one donor that might play important roles in maintaining the homeostasis of the human body. We have made the AHCA publicly available (http://research.gzsums.net:8888), as a resource to uncover key events during the development of human disease in the context of heterogeneity of cells and organs.

It has been demonstrated that $\mathrm{T}_{\mathrm{N}}$ cells are generated in the thymus and populate lymphoid tissues where they differentiate to $T_{\mathrm{EFF}}$ cells upon antigen stimulus, and subsequently develop into long-lived memory $\mathrm{T}$ cells [54]. However, how $\mathrm{T}$ cells develop into different states throughout human organs and the links across $\mathrm{T}$ cells of different states as well as the underlying regulatory networks are largely unknown, especially in the context of one individual body. In our study, trajectory analysis revealed a clear development route from $\mathrm{T}_{\mathrm{N}}$ cells into $\mathrm{T}_{\mathrm{EFF}}$ cells and then $\mathrm{CD} 4^{+}$and $\mathrm{CD}^{+} \mathrm{T}_{\mathrm{RM}}$ cells in non-lymphoid organs with terminal developmental states, which is consistent with 
previous findings [54]. Indeed, $\mathrm{T}_{\mathrm{RM}}$ cells share many properties with recently activated effector $\mathrm{T}$ cells, supporting the fact that they may constitute a terminally differentiated population [55-57]. However, for $\mathrm{CD} 4^{+} \mathrm{T}$ cells, we noted a clear $\mathrm{T}_{\mathrm{CM}}$ cell cluster (STMN1_ $\mathrm{T}_{\mathrm{CM}}$ ) at the end of the trajectory, likely differentiated from $\mathrm{T}_{\mathrm{RM}}$ cells (TNF $\left.\mathrm{T}_{\mathrm{RM}}\right)$. Together with STMN1_ $\mathrm{T}_{\mathrm{CM}}$ cells collected from lymph nodes, this observation provides a clue to solving the puzzle as to whether $\mathrm{T}_{\mathrm{RM}}$ cells can further differentiate or migrate back to the lymphoid compartment [58]. In support of such, a very recent mice model study demonstrated that $\mathrm{T}_{\mathrm{RM}}$ cells in the skin could differentiate into $\mathrm{T}_{\mathrm{CM}}$ and $\mathrm{T}_{\mathrm{EM}}$ cells upon local reactivation, which then rejoined the circulation. Moreover, trajectory analysis with $\mathrm{TNF}_{-} \mathrm{T}_{\mathrm{RM}}$ and $\mathrm{STMN} \mathrm{T}_{\mathrm{CM}}$ clusters revealed that $\mathrm{TNF}_{-} \mathrm{T}_{\mathrm{RM}}$ cells from two branches in an early state gradually progressed towards STMN1_ $\mathrm{T}_{\mathrm{CM}}$ cells in a terminal state (Additional file 1: Figure S28A). $\mathrm{T}_{\mathrm{RM}}$ cells at the beginning of the trajectory expressed high levels of $\mathrm{T}_{\mathrm{RM}}$ markers, such as RUNX3, NR4A1, chemokines (CCL5) [59], and other $\mathrm{T}_{\mathrm{RM}}$ associated genes, including ID2 [60]. By contrast, $\mathrm{T}_{\mathrm{CM}}$ cells at the end of the trajectory were marked with expression of well-known $\mathrm{T}_{\mathrm{CM}}$ molecules, such as SELL and CCR7 (Additional file 1: Figure S28B). Taken together, these observations suggest that $\mathrm{T}_{\mathrm{RM}}$ cells have developmental plasticity rather than representing a terminal stage of differentiation [61]. We also noted that $\mathrm{T}_{\mathrm{RM}}$ cells exhibit development states at organ-specific patterns and consistently these cells were regulated by different types of TFs, suggesting that tissue microenvironments might regulate gene expression by affecting TF's activity, which in turn shapes specific $\mathrm{T}$ cell phenotypes. TCR analysis tracking cell of a same lineage revealed widespread links among subpopulations of $\mathrm{T}_{\mathrm{RM}}$ cells; however, these are considered as non-recirculating [62]. Moreover, intensive sharing of TCR was observed among $\mathrm{T}_{\mathrm{RM}}, \mathrm{T}_{\mathrm{EM}}$, and $\mathrm{T}_{\mathrm{EFF}}$ cells (Additional file 1: Figure S14I, J). Together with their development states, these results suggest that $\mathrm{T}_{\mathrm{EM}}$ cells and $\mathrm{T}_{\mathrm{EFF}}$ cells might enter the tissues and develop into $\mathrm{T}_{\mathrm{RM}}$ cells and IEL $\mathrm{T}$ cells. In addition, we observed the branching out of CTLA4_ $\mathrm{T}_{\text {reg }}$ cells next to KLF2_ $T_{C M}$ cells along the development trajectory from $T_{N}$ cells to $T_{R M}$ cells (Fig. 2e). Given that previous in vitro studies have demonstrated a potential development from central memory $\mathrm{T}$ cells to $\mathrm{T}_{\text {reg }}$ cells upon stimulation [63, 64], this observation suggested that differentiation of $\mathrm{T}_{\text {reg }}$ cells from central memory $\mathrm{T}$ cells is possible. However, whether it holds true in vivo remains to be established with precise lineage tracing methods.

Infiltrating macrophages come from classical monocytes in pathological settings, such as cancers [65], while various origins of adult macrophages among tissues in a steady state have been reported [66]. As such, it has been debated how macrophages are renewed in the maintenance of hemostasis in tissues, whether through local proliferation or recruitment of monocytes from peripheral blood [67, 68]. In our study, multiple observations suggest that macrophages in organs are derived from either circulating monocytes or in situ expansion and proliferation of macrophage populations coping with the local microenvironments. Consistently, entry of monocytes to steady-state non-lymphoid organs and self-maintenance of tissue macrophages have been reported in mice models [36, 69]. Although our observations suggest a potential developmental relationship between circulating monocytes and tissue-resident macrophages, we acknowledge that further investigations are needed to address whether self-expansion alone, or slightly together with circulating monocytes contributes to the development 
of tissue-resident macrophages, considering that most tissue-resident macrophages have been demonstrated to be originated from embryonic progenitor cells [36].

We observed a more isolated clustering of epithelial and stromal cells compared with immune cells, which have circulating capability. Because epithelial and stromal cells are fundamental components that form protective barriers and supporting matrix for many organs, disruptions in their homeostasis have been implicated in various diseases [7073]. Accumulating studies have demonstrated the remarkable functional heterogeneity of epithelial cells among tissues [74-76]. Consistently, our GO enrichment analyses revealed very diverse functions of epithelial cells among different tissues, as well as stromal cells. Taken together, a higher degree of heterogeneity might reflect a higher degree of terminal differentiation states and distinct specific functions of these cells among different organs. Nevertheless, we note that epithelial cells derived from digestive organs had similar biological functions and activation of TFs, which were consistently observed in both our AHCA and the HCL datasets [20]. Given that most of the digestion-related organs develop from the endoderm, this might explain their similarity in genetic profiles and functions [77]. In addition, we suspected that the epithelial cells with the same digestive functions might also share similar responses to pathogens or stimuli [78].

The AHCA not only brings more detailed understanding of cell development and heterogeneity, but also reveals novel cell types and genes as well as regulatory factors that might be important for cell development. We identified subsets of novel cells, including $\mathrm{COCH}^{+}$fibroblasts and FibSmo cells with a broad distribution among organ tissues. TFs are known as the "master regulators" for gene expression [79, 80]. We identified numerous novel TFs in regulating the development of different cell states of major cell types, such as CEBPD, EGR1 in CD4 ${ }^{+} \mathrm{T}_{\mathrm{RM}}, E L F 1$ in $\mathrm{CD} 8^{+} \mathrm{T}_{\mathrm{RM}}$, MAFF in both $\mathrm{CD} 4^{+}$and $\mathrm{CD} 8^{+} \mathrm{T}_{\mathrm{RM}}, P O L R 2 A$ in B cells, and KLF13 in plasma cells, as well as $E G R 1, M Y C, Y Y 1$, and BCL11A in the non-digestive tissues. These findings not only extend our understanding in how the TFs regulate gene expression and shape different phenotypes, but also provide potential gene combinations in reprogramming applications. The AHCA also provides useful data to explore the cellular networks at a singlecell resolution. We discovered a large number of interactions between immune cells and other cells in all tissues, reflecting essential and broad communications between immune cells and other cell types in the human body. Epithelial cells had the most frequent inter-cell interactions compared with other cell types, suggesting that they could interact with each other intensively to regulate their biological functions (Additional file 1: Figure S29). Moreover, the AHCA is based on a large scale of single-cell transcriptomes from multiple organs, which might provide common biological understanding at a higher resolution. First, the heterogeneous nature of human cells in organs is consistent with the findings in mice [8] and a recent study with human tissues [20]. Second, we identified well-known markers for different cell types and well-characterized TFs responsible for cell development, such as TCF7, SELL, MYC, and KLF2 for $\mathrm{T}_{\mathrm{N}}$ cells and TBX21, STAT1, and IRF1 for $\mathrm{T}_{\mathrm{EFF}}$ cells. Third, similar transcription profiles of welldifferentiated epithelial cells were observed between the AHCA and the recent published HCL datasets [20] (Fig. 5a, Additional file 1: Figure S18B), as were important TFs regulating plasma cell development (Fig. 3e, Additional file 1: Figure S16F) and digestion-related cells (Fig. 5g, Additional file 1: Figure S20B). Lastly, our discovery of novel and rare cell 
types was validated in existing datasets and replicated in independent human samples (Additional file 1: Figure S4-S10).

Cellular dissociation is a prerequisite of technical manipulation in single-cell studies. One previous study reported dissociation procedures induced stress and caused transcriptional disturbances of varying degrees, leading to a misinterpretation of results [81]. Enzymes and duration of dissociation procedures might be two important factors [81, 82]. Considering the properties of different enzymes and the heterogeneous cell types in organs, we optimized the protocols to achieve better dissociation and higher cell viability for each organ (Additional file 11: Table S49). We observed that most organs had a higher density of low total dissociation scores [81], meaning that dissociation-related genes were not significantly induced in the majority of cells (Additional file 1: Figure S30). Moreover, high expression of FOS, a dissociation-related gene according to previous studies [81], was observed widely in multiple human tissues before dissociation (Additional file 1: Figure S31). Furthermore, each major cell type derived from multiple organs treated with different dissociation procedures shared similar transcriptional profiles as reflected by the cluster analyses. These suggest that the dissociation procedures had minimal effects on the transcriptomes in our study. However, we could not rule out the possibility that the dissociation procedures might have impacts on some cell types, which awaits further investigations.

We acknowledge that the current AHCA has several limitations. First, although we obtained a sufficient number of sequencing reads for each sample, the number of genes detected in each cell was limited. This might underestimate the roles of some lowly expressed genes, such as long non-coding RNAs. Second, we obtained around 5000 cells on average for each organ, which might limit our ability to identify rare cell types and thus underestimate the heterogeneity of inter-cell interactions in organs. Third, we included only 15 organ tissues from a single donor in our study. Further studies on gene expression profiling at both the transcriptional and protein levels, as well as functional characterization with more organs from a larger number of donors, would provide a much broader and more detailed global view of the human cell atlas and cell biology.

\section{Conclusions}

We generated an AHCA, by profiling the single-cell transcriptome for more than 84, 000 cells of 15 organs from one research-consented donor. The AHCA uncovered the heterogeneity of cells in major human organs, containing more than 250 subtypes of cells. Comprehensive analyses of the AHCA enabled us to delineate the developmental trajectories of major cell types and to identify novel cell types, regulators, and key molecular events that might play important roles in maintaining the homeostasis of the human body and/or those otherwise developing into human diseases.

\section{Materials and methods}

\section{Organ tissue collection}

An adult male donor who died of a traumatic brain injury was recruited at the First Affiliated Hospital of Sun Yat-sen University (SYSU-1H). For single-cell RNA sequencing, besides the organs for transplantation purposes, we collected tissues from 15 organs in 
sequence, including the blood, bone marrow, liver, common bile duct, lymph node (hilar and mesenteric), spleen, heart (apical), urinary bladder, trachea, esophagus, stomach, small intestine, rectum, skin, and muscle (thigh). All hollow viscera tissues were dissected according to the whole layer structure, and all parenchymal viscera tissues were obtained from the organ lower pole. All the tissue collection procedures were accomplished within 20 min to maximize cell viability. To avoid cross-contamination, we used different sets of sterilized surgical instruments. The blood and bone marrow samples were loaded into 10-ml anticoagulation tubes containing EDTA (BD Biosciences, Cat. no. BD-366643), and other tissues were placed in physiological saline $\left(4^{\circ} \mathrm{C}\right)$ to wash away the blood and secretions, and then immediately in a D10 resuspension buffer, containing a culture medium (DMEM medium; Gibco ${ }^{\mathrm{m}}$, Cat. no. 11965092) with $10 \%$ fetal bovine serum (FBS; Gibco ${ }^{\mathrm{Tm}}$, Cat. no. 10099141). All tissue samples were kept on ice and delivered to the laboratory within $40 \mathrm{~min}$ for further processing. For immunohistochemistry assays, paraffin-embedded normal samples were collected from additional patient donors at the SYSU-1H.

\section{Tissue dissociation and cell purification}

All tissues were dissociated within $1.5 \mathrm{~h}$, and viable cells were collected at the end using fluorescence-activated cell sorting (FACS; BD FACS Aria ${ }^{\mathrm{Tm}}$ III). For solid tissues exclusive of the liver, each fresh tissue was cut into 1-mm pieces and incubated with a proper digestive solution including enzyme cocktail (Additional file 11: Table S49), followed by neutralization with the D10 buffer and then passed through a $40-\mu \mathrm{m}$ cell strainer (BD, Cat. no. 352340). The cell suspension was centrifuged at $300 \times g$ for $5 \mathrm{~min}$ at $4{ }^{\circ} \mathrm{C}$, and the pellet was resuspended with a $0.8 \% \mathrm{NH}_{4} \mathrm{Cl}$ (Sigma-Aldrich, Cat. no. 254134-5G) red blood cell lysis buffer (RBCL) on ice for $10 \mathrm{~min}$, followed by an additional wash with the D10 buffer. The liver tissue was cut into 3-4-mm pieces and incubated with 1 mM EGTA (Sigma-Aldrich, Cat. no. E0396-10G) in 1× DBPS (Gibco ${ }^{\mathrm{ms}}$, Cat. no. 14190250) for $10 \mathrm{~min}$ at $37^{\circ} \mathrm{C}$ with rotation at $50 \mathrm{rpm}$. After washing with $1 \times$ DPBS to remove EGTA, each tissue was then incubated in a pre-warmed digestion buffer (Additional file 11: Table S49) with rotation at $100 \mathrm{rpm}$ at $37^{\circ} \mathrm{C}$ for $30 \mathrm{~min}$. The liver cell suspension was carefully passed through a $70-\mathrm{mm}$ nylon cell strainer (BD, Cat. no. 352350), which was further centrifuged at $50 \times g$ for $3 \mathrm{~min}$ at $4{ }^{\circ} \mathrm{C}$ to pellet hepatocytes. The supernatant was centrifuged at $300 \times g$ for $5 \mathrm{~min}$ at $4{ }^{\circ} \mathrm{C}$ to pellet nonparenchymal cells. The pellet was resuspended and treated with RBCL. The blood and bone marrow samples were pelleted by centrifugation at $300 \times g$ for $5 \mathrm{~min}$ at $4{ }^{\circ} \mathrm{C}$ and resuspended with RBCL on ice for $10 \mathrm{~min}$, followed by an additional wash with the D10 buffer. All cells from each tissue were resuspended with the D10 buffer to a concentration of 50-500 million cells per milliliter and stained with Calcein AM (component A: AM) and Ethidium homodimer-1 (component B: EH) in LIVE/DEAD Viability/Cytotoxicity Kit (Invitrogen, Cat. no. L3224) for 20 min on ice. Only the $\mathrm{AM}^{+} \mathrm{EH}^{-}$cells were collected by FACS for each tissue.

\section{cDNA library preparation}

The concentration of single-cell suspension was determined using a Cellometer Auto 2000 instrument (Cellometer) and adjusted to 1000 cells $/ \mu$ l. Approximately 14,000 cells 
were loaded into a CHROMIUM instrument (10x Genomics, CA, USA) according to the standard protocol of the Chromium single cell V(D)J kit in order to capture $5000 \sim 10,000$ cells per channel. In brief, mRNA transcripts from each sample were ligated with barcoded indexes at 5 '-end and reverse transcribed into cDNA, using GemCode technology (10x Genomics, USA). cDNA libraries including the enriched fragments spanning the full-length V(D)J segments of $\mathrm{T}$ cell receptors (TCR) or B cell receptor (BCR), and $5^{\prime}$-end fragments for gene expression were separately constructed, which were subsequently subjected for high-throughput sequencing.

\section{Single-cell RNA sequencing data processing}

5 '-end CDNA, TCR, and BCR libraries were mixed and subjected for sequencing on Illumina HiSeq XTen instruments with paired-end $150 \mathrm{bp}$. Raw data (BCL files) from HiSeq platform was converted to fastq files using Illumina-implemented software bcl2fastq (version v2.19.0.316). cDNA reads were aligned to the human reference genome (hg38), and digital gene expression matrix was built using STAR algorithm in CellRanger ("count" option; version 3.0.1; 10x Genomics) [83]. TCR and BCR reads were aligned to human reference VDJ dataset (http://cf.10Xgenomics.com/supp/cell-vdj/ refdata-cellranger-vdj-GRCh38-alts-ensembl-2.0.0.tar.gz) using CellRanger ("vdj" option; version 3.1.0; 10x Genomics). Parameters were set as default except for "forcecells" as 13,000. Raw digital gene expression matrix in the "filtered_feature_bc_matrix" file folder generated by CellRanger was used for further analysis.

\section{Cell clustering, doublet identification, and differential gene expression analysis}

Quality control filtering, variable gene selection, dimensionality reduction, and clustering for cells were performed using the Seurat package [6] (version 3.1.5; https://satijalab.org/seurat). "DoubletFinder" (version 2.0.3; https://github.com/chris-mcginnis-ucsf/DoubletFinder) was used to identify doublets in each organ. All the analytic packages were performed in $\mathrm{R}$ software (version 3.6.3; https://www.r-project.org), with default settings unless otherwise stated. For each tissue, output cells were forced to 13,000 under "cellranger count" module, and we removed cells with low quality (UMI $<1000$, gene number $<$ 500 , and mitochondrial genome fragments $>0.25$ ) as well as genes with rare frequencies $(0.1 \%$ of all cells). For the remaining cells, gene expression count data for each sample was normalized with "NormalizedData" function, followed by scaling to regress UMIs and mitochondrial content using "ScaleData" function (negative binomial model). Principal component analysis (PCA) and t-SNE implemented in the "RunPCA" and "RunTSNE" functions, respectively, were used to identify the deviations among cells. Genes with high variations were identified using "FindVariableGenes" and included for PCA ("mean.cutoff" $\geq 0.1$, "dispersion.cutoff" $\geq 0.5$ ). We used a different value of perplexity and the number of principal components (PCs) determined by elbow plots for each tissue and cell type (Additional file 11: Table S50). Cell clusters were identified using the "FindClusters" function and shown using t-SNE. Subsequently, "DoubletFinder" was used to identify doublets using the same PCs in PCA above, assuming the $5 \%$ doublet formation rate to the loaded cells for each sample in a droplet channel. The optimal $\mathrm{pK}$ values were determined for each organ based on the mean-variance normalized bimodality coefficient (BCmvn; Additional file 11: Table S51). 
After doublet removal, we rerun the above analyses. Next, differential expression markers or genes were determined using the Wilcoxon test implemented in the "FindAllMarkers" function, which was considered significant with an average natural logarithm (fold change) of at least 0.25 and a Bonferroni-adjusted $P$ value lower than 0.05 . Subsequently, the candidate markers were reviewed and were used to annotate cell clusters. We further manually removed cell clusters that had multiple well-defined marker genes and overlapped gene profiles of multiple different cell types (Additional file 2: Table S4-S18). For analyses of the merged data from all tissues, we used 30 PCs and a resolution parameter set to 1 for cell clustering.

\section{Identification and removal of highly transcribed genes with contamination potentials}

Because we observed that some cell-specific genes were broadly expressed among all cell types in a tissue, for example, APOC3 in enterocytes cells in the small intestine with an average of more than 200 UMIs in a cell, we suspected that if a fraction of a certain type of cells were broken during the sample processing, cell-specific genes with high transcriptions would be released and thus contaminate all cell droplets. Especially, as such the genes would screw the differential expression analysis of cell type among all tissues. Therefore, we identified these genes and removed them for comparison analyses within major cell types. We assumed that non-epithelial cells from a same linage have similar gene profiles at a certain degree and that a few genes would have modest expression in only one organ. Here is a given example for $\mathrm{T}$ cells, in which we grouped together the cells previously labeled with NK/T, T, and immune cells (Additional file 2: Table S4-S18) in each tissue. Next, we determined the top $2 \%$ of genes with high transcription in each tissue sample based on the total number of UMIs (Additional file 11: Table S52) and marked these as potentially contaminating genes. We further randomly sampled 300 cells for each cell type in a tissue and as such generated artificial data for all tissues, with which differential expression gene analysis was performed using the "FindAllMarker" function. Any gene with a normal logarithm of FC above 0.25 and with an expression in less than $5 \%$ cells in all other tissue samples was considered a contaminating gene. We performed the above sampling and calculation for four independent times, with seed numbers 1 to 4 in the "FindMarker" function, and only the genes commonly observed in the four calculations were determined as contaminating genes. After removing the above contaminating genes, we performed cluster analysis with the first 20 PCs and a resolution of 1.5. After further removal of cell clusters that had multiple well-defined marker genes of different cell types, we repeated cluster analysis using a lower resolution setting and removing the genes encoding immunoglobulins from the gene expression matrices (Additional file 11: Table S50) to identify $\mathrm{CD} 4^{+}, \mathrm{CD}^{+}$, and NK cell (Additional file 11: Table S53) clusters. For B cells, plasma cells, endothelial cells, macrophages, monocytes, and fibroblasts, we applied a similar strategy to remove contaminating genes (Additional file 11: Table S54S58) and cell clusters with multiple cell-specific markers.

\section{Trajectory analysis}

We performed trajectory analysis using Monocle3 alpha [4] for all tissue-derived T cells, macrophages/monocytes, according to the general pipeline (http://cole-trapnell-lab. github.io/monocle-release/monocle3/). For $\mathrm{T}$ cells, after identification of $\mathrm{T}$ cells clusters 
for $\mathrm{CD}^{+}$and $\mathrm{CD} 8^{+} \mathrm{T}$, raw gene expression counts of cells were imported to the software. Only genes matching the thresholds (both of mean expression and dispersion ratio greater than 0.15 for $\mathrm{CD}^{+}, \mathrm{CD}^{+} \mathrm{T}$ cell and myeloid cells) were used for cell ordering and training the pseudo-time trajectory. For investigating the dynamic gene expression between $\mathrm{T}_{\mathrm{RM}}$ and $\mathrm{T}_{\mathrm{CM}}$, we extracted those two $\mathrm{CD} 4^{+} \mathrm{T}$ clusters and performed the trajectory analysis using Monocle2 with 1000 high variable genes. For trajectory analysis of intestinal macrophages/monocytes, we extracted the cell clusters with more than 50 cells in our dataset and combined 50 embryonic macrophages from a previous study [39]. The analysis was done by correcting the batch effect using function "align_cds" of Monocle3 with 25 PCs included.

\section{TCR and BCR analysis}

We assessed the enrichment of TCR and BCR in various organs using R package STAR TRAC (version 0.1.0) [23], which included only the cells with the certain clonotypes assigned by CellRanger (version 3.1.0 with updated algorithms to improve the identification of TCR/BCR clonotypes) and with paired chains ( $\alpha$ and $\beta$ for T cells, heavy and light chains for B cells). In brief, cells sharing identical TCR or BCR clones between tissues were measured using migration-index score, and the degree of cell linking between different clusters of $\mathrm{T}$ cells or $\mathrm{B}$ cells was determined by the transition-index score. TCR or BCR diversity (Shannon-index score) was calculated using " 1 - expansionindex score.” For the detailed pipeline, please refer to the website (https://github.com/ Japrin/STARTRAC/blob/master/vignettes/startrac.html).

\section{Presenting-antigen score}

To evaluate the antigen-presenting ability of extra- and intracellular of each cell, antigen-presenting score (APS) was calculated using the "AddModuleScore" function implemented in the Seurat package, with gene sets "MHC_CLASS_II_ANTIGEN_ PRESENTATION" and "REACTOME_CLASS_I_MHC_MEDIATED_ANTIGEN_PROCESSING_PRESENTATION" pathways, respectively, in the REACTOME database (http://www.reactome.org).

\section{Gene Ontology pathway enrichment analysis}

All GO enrichment analysis was performed using the online tools metascape [84] with the "multiple gene list mode" (http://metascape.org/gp/index.html). For epithelial cells, we selected genes with $\mathrm{FC} \geq 2$ and p.adjust $<0.05$ for each tissue, while $\mathrm{FC} \geq 1.5$, p.adjust $<0.05$, and ptc. $1>0.2$ for each cluster. For non-epithelial cells, we selected genes with $\operatorname{lnFC} \geq 0.25$, p.adjust $<0.05$, and ptc. $1>0.2$ in each cluster. Only the genes that ranked in the top 150 according to the FC were used for comparisons in each tissue and subpopulation. The background was given as all the genes expressed in corresponding cell types. In addition, only the gene sets in the "GO Biological Processes" were considered.

\section{Cellular interaction analysis}

To investigate the cellular interaction, we identified the inferred paired molecules using CellphoneDB software (version 2.0) [18] with default parameters. First, to facilitate the 
pairwise analyses by reducing cell types, we grouped clusters within major cell types, including T, B, NK, myeloid, endothelial cells, fibroblasts, smooth muscle cells, and FibSmo cells within each tissue. Moreover, we manually assigned ITGB1_ $\mathrm{T}_{\mathrm{N} / \mathrm{CM}}$, LEF1_ $\mathrm{T}_{\mathrm{N} / \mathrm{CM}}$, and GADD45B_ $\mathrm{T}_{\mathrm{N} / \mathrm{CM}}$ as $\mathrm{T}_{\mathrm{CM}}$ cell cluster, and $\mathrm{KLF} 2 \mathrm{~T}_{\mathrm{N} / \mathrm{CM}}$ as $\mathrm{T}_{\mathrm{N}}$ cell cluster based on their expression profiles and the developmental states along the trajectory trees, although naïve and memory $\mathrm{T}$ cell clusters could not be accurately determined in our study. The grouped cell types were as follows: CD4 $4^{+} \mathrm{T}$ cell $\left(\mathrm{T}_{\mathrm{N}}, \mathrm{T}_{\mathrm{CM}}, \mathrm{T}_{\mathrm{reg}}, \mathrm{T}_{\mathrm{EM}}\right.$, $\mathrm{T}_{\mathrm{h} 1}$, and $\left.\mathrm{T}_{\mathrm{RM}}\right), \mathrm{CD} 8^{+} \mathrm{T}$ cell $\left(\mathrm{T}_{\mathrm{N}}, \mathrm{T}_{\mathrm{CM}}, \mathrm{T}_{\mathrm{EM}}, \mathrm{T}_{\mathrm{EFF}}, \mathrm{T}_{\mathrm{RM}}\right.$, IEL, and MAIT), $\gamma \delta \mathrm{T}$ cell, $\mathrm{B}$ cell (naïve and memory B cell), NK cell, plasma cell, myeloid cell (monocyte: Mon, macrophage: Mac, and dendritic cell: DC), endothelial cell (BEC and LEC), fibroblast (Fib), smooth muscle cell (Smo), and FibSmo cell (FibSmo). We did not merge subpopulations of epithelial cells because of their high degree of heterogeneity in each tissue. Considering the test efficiency and computational burden, we focused on cell types with more than 30 cells and only randomly selected 250 cells of each cell type for analysis in each tissue. The significant ligand-receptor pairs were filtered with a $P$ value of less than 0.05 and an average expression of interacting pairs larger than 0 . All the analyses above were performed as tissue independent. For the analysis of interaction across organs, we only calculated between any one of the immune cells in a tissue and its interacting cells from a different organ. Visualization of interaction network was done using Cytoscape (version 3.7.0).

\section{Single-cell regulatory network inference and clustering analysis}

We conducted SCENIC analysis on cells passing the quality controls for each major cell types, using R package SCENIC (version 1.1.3) as previously described [85]. Regions for TF searching were restricted to $10 \mathrm{k}$ distance centered the transcriptional start site (TSS) or $500 \mathrm{bp}$ upstream of the TSSs. Transcription factor binding motifs (TFBS) overrepresented on a gene list and networks inferring were done using $\mathrm{R}$ package RcisTarget (version 1.6.0) and GENIE3 (version 1.8.0), respectively, with the 20-thousand motifs database. We randomly selected no more than 250 cells for each cell cluster. The input matrix was the normalized expression matrix from Seurat. The clusterspecific TFs of one cluster were defined as the top 10 or 15 highly enriched TFs according to a decrease in fold change compared with all the other cell clusters using a Wilcoxon rank-sum test.

\section{Validation analysis in existing datasets}

For B and epithelial cells in the HCL dataset [20], we applied similar procedures for cell clustering and differential gene expression analyses as described above. We only extracted adult B and epithelial cell clusters identified by the HCL dataset, considering high sequencing coverage and with less potential cross-cell contamination compared with the other cell types. Genes encoding immunoglobulin were removed in the epithelial cells from raw count data before further analysis. We set the "mean.cutoff" and "dispersion.cutoff" as 0.05 and 0.2 in "FindVariableFeatures" step for both B and epithelial cell analyses.

For validation of $\mathrm{COCH}^{+}$fibroblasts [86-88], sweat gland cells [86, 87], and FibSmo cells $[20,89]$, cells from each individual dataset were merged and batch effect was 
removed using the "FindIntegrationAnchors" and "IntegrateData" functions in the Seurat package. The downstream analyses followed the same pipeline of our AHCA dataset.

\section{Calculation of dissociation scores}

For each organ, principal component analysis was performed on a subset of 140 human homologous dissociation-related genes as described previously [81]. The first principal component was used as the "dissociation score" as it corresponds to the variance within these genes.

\section{Analysis of differential pathway}

Gene set variation analysis (GSVA) or gene set enrichment analysis (GSEA) was performed to identify significantly enriched genes in each transcriptional dataset, using $\mathrm{R}$ package GSVA (version 1.34.0) or GSEA software (version 4.0.3) (https://www.gsea-msigdb.org/ gsea/index.jsp) on the 50 hallmark pathways with default parameters, respectively. For epithelial cells in AHCA and HCL datasets, GSVA was performed on the 50 hallmark pathways and additional curated metabolic pathway dataset [90].

\section{Immunofluorescence staining assay}

For immunofluorescence staining assay, tissue samples were collected within $20 \mathrm{~min}$, washed with $1 \times$ DPBS, fixed in $4 \%$ paraformaldehyde (pH 7.0), and embedded in paraffin according to routine methods. These paraffin blocks were cut into $4-\mu \mathrm{m}$ slides and adhered on the slide glass. The sections were deparaffinized, rehydrated, and subjected to blockade of endogenous peroxidase activity with $3 \% \mathrm{H}_{2} \mathrm{O}_{2}$, and high-temperature antigen retrieval. The tissues were incubated with 3\% BSA at room temperature for 30 min, and then incubated overnight at $4{ }^{\circ} \mathrm{C}$ with the primary detection antibodies for different organs (Additional file 11: Table S59). The slides were then incubated with the secondary antibody (HRP polymer, anti-rabbit $\operatorname{IgG}$ ) at room temperature for $50 \mathrm{~min}$. Subsequently, fluorophore (tyramide signal amplification, TSA plus working solution; Servicebio, Cat. no. G1222/3/4) was applied to the tissues. The slides were microwave heat-treated after each TSA treatment, and the primary antibodies were applied sequentially for different organs, followed by incubation with the secondary antibody and TSA treatment. Nuclei were stained with 4' -6' -diamidino-2-phenylindole (DAPI; Invitrogen, Cat. no. D1306) after all the antigens had been labeled. Negative controls were performed using similar procedure above, except for replacing the primary antibody with $1 \times$ DPBS. To obtain multispectral images, slides were scanned using the Pannoramic MIDI II system (3DHISTECH, Hungary).

\section{Supplementary Information}

The online version contains supplementary material available at https://doi.org/10.1186/s13059-020-02210-0.

Additional file 1: Figure S1. Quality information of single-cell RNA sequencing, related to Fig. 1. Figure S2. Distribution of cell cluster in each organ tissue, related to Fig. 1. Figure S2. Distribution of cell cluster in each organ tissue (continued). Figure S3. The expression of marker genes and distribution of sweat gland epithelial cells and a novel fibroblast subtype, related to Fig. 1. Figure S4. Expression of DCD, SCGB2A2, PIP, KRT19, and MUCL1 genes in skin cells derived from public datasets, related to Fig. 1. Figure S5. Expression of COCH and MMP2 in cells from multiple human samples from public datasets, related to Fig. 1. Figure S6. Gene signature of $\mathrm{COCH}^{+}$fibroblasts in skin, related to Fig. 1. Figure S7. Expression of marker genes in cells from bladder samples derived from public datasets, related to Fig. 1. Figure S8. Representative images of immunofluorescence staining of sweat gland 
epithelial cells in skin samples. Figure S9. Representative images of immunofluorescence staining of $\mathrm{COCH}^{+}$fibroblasts in the skin and heart tissues. Figure S10. Representative images of immunofluorescence staining of FibSmo cells in the bladder, rectum, and heart tissues. Figure S11. Distribution of cell clusters in different organs, related to Fig. 1. Figure S12. Comparisons of cell-type determinations by whole single-cell transcriptome dataset and manual annotation of each organ, related to Fig. 1. Figure S13. The heterogeneity and developmental trajectory analysis of T cells, related to Fig. 2. Figure S14. The heterogeneity and clonalities of T cell clones in human body, related to Fig. 2. Figure S15. The heterogeneity and clonalities of T cell clones in the human body, related to Fig. 2 . Figure S16. The heterogeneity of B and plasma cells, related to Fig. 3. Figure S17. Heterogeneity and trajectory analysis of macrophages, related to Fig. 4. Figure S18. The gene expression heterogeneity of epithelial cells among inter- and intra-organ tissues, related to Fig. 5. Figure S19. Expression profiles and altered pathways in digestive and non-digestive epithelial cell clusters. Figure S20. The antigen-presenting heterogeneity and regulons of epithelial cells among inter- and intra-organ tissues, related to Fig. 5. Figure S21. The heterogeneous expression pattern of endothelial cells. Figure S22. The similarity and heterogeneity of fibroblasts and smooth muscle cells. Figure S23. Gene expression and biological functions of fibroblasts, smooth muscle and FibSmo cells. Figure S24. Overview of the ligand-receptor interactions between different cell types, related to Fig. 6. Figure S25. Overview of the ligand-receptor interactions between epithelial and myeloid cells, related to Fig. 6. Figure S26. Overview of the ligand-receptor interactions between $C D 8^{+} T$ and myeloid cells, related to Fig. 6. Figure S27. Overview of the ligand-receptor interactions between $\mathrm{CD}^{+} \mathrm{T}$ and stromal cells (fibroblast, smooth muscle cell, and FibSmo cell), related to Fig. 6. Figure S28. Pseudotime trajectory analysis and gene expression profiles of TNF_ $T_{\text {RM }}$

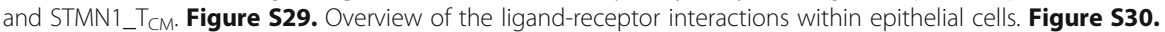
Histogram plots of the density of dissociation scores for each organ. Figure S31. Representative images of immunofluorescence staining of FOS and CD8A human tissue samples.

Additional file 2: Table S1. Basic information of sequencing, related to Fig. 1. Table S2. Cell number, the median of UMI and genes detected in each organ, respectively, related to Fig. 1. Table S3. Marker genes and related references. Table S4-S18. List of markers information (top 50) for each cell type in the 15 organs, related to Fig. 1. Table S19. List of markers information (top 50) for each cell type in the merged dataset, related to Fig. 1. Table S20. Cell counts in each organ for each cluster indicated in Figure S11.

Additional file 3: Supplementary Notes. This supplementary material includes a detailed description for the validation of sweat gland epithelial cells, $\mathrm{COCH}^{+}$fibroblasts, and FibSmo cells in existing datasets, and the evidence showing that the expression of HSPATA, FOS, and JUN in the CD8 ${ }^{+} T$ cells are unlikely stress-induced artefacts.

Additional file 4: Table S21. Distribution of major cell types in each organ, related to Fig. 2. Table S22. List of marker information (top 50) for each subpopulation of CD4 ${ }^{+} \mathrm{T}$ cells, related to Fig. 2. Table S23. List of marker information (top 50) for each subpopulation of $\mathrm{CD}^{+} \mathrm{T}$ cells, related to Fig. 2. Table S24. List of TFs information for each subpopulation of $\mathrm{CD}^{+} \mathrm{T}$ cell, related to Fig. 2. Table S25. List of TFs information for each subpopulation of $\mathrm{CD}^{+} \mathrm{T}$ cells, related to Fig. 2. Table S26. Detailed information of CD4 ${ }^{+} \mathrm{TCR}$ repertoire, related to Fig. 2. Table S27. Detailed information of $\mathrm{CD}^{+}$TCR repertoire, related to Fig. 2.

Additional file 5: Table S28. List of marker information for each subpopulation of B and plasma cells in AHCA dataset, related to Fig. 3. Table S29. List of TFs information for each B and plasma cells subpopulation in AHCA dataset, related to Fig. 3. Table S30. List of marker information for each subpopulation of B and plasma cells in HCL dataset, related to Fig. 3. Table S31. List of TFs information for each subpopulation of B and plasma cells in HCL dataset, related to Fig. 3. Table S32. Detailed information of BCR repertoire, related to Fig. 3.

Additional file 6: Table S33. List of marker information (top 50) for each subpopulation of myeloid cells, related to Fig. 4. Table S34. List of TFs information for each myeloid cell subpopulation, related to Fig. 4.

Additional file 7: Table S35. List of marker information for epithelial cells of each organ in AHCA dataset, related to Fig. 5. Table S36. Cell counts in each organ for each cluster indicated in Fig. 5C in AHCA dataset. Table S37. List of marker information (top 50) of each subpopulation of epithelial cells in AHCA dataset, related to Fig. 5. Table S38. Marker genes and related references for HCL epithelial cells. Table S39. Cell counts in each organ for each cluster indicated in Figure S18E in HCL dataset. Table S40. List of marker information (top 50) of each subpopulation of epithelial cells in HCL dataset, related to Fig. 5. Table S41. List of TFs information for each subpopulation of epithelial cells in AHCA dataset, related to Fig. 5. Table S42. List of TFs information for each subpopulation of epithelial cells in HCL dataset, related to Fig. 5.

Additional file 8: Table S43. List of marker information (top 50) for each endothelial cell cluster. Table S44. List of marker information (top 50) for each fibroblast, smooth muscle and FibSmo cell cluster. Table S45. List of marker information for fibroblast, smooth muscle and FibSmo cell.

Additional file 9: Table S46. Frequency of potential interacting pairs, related to Fig. 6. Table S47. Detailed information of interacting pairs in each tissue related to Fig. 6.

Additional file 10: Table S48. Detailed information of interacting pairs across tissues, related to Fig. 6

Additional file 11: Table S49. The digestion protocols for each organ. Table S50. The PCs and resolution used for clustering of each organ or major cell type. Table S51. Optimal pK values for each organ. Table S52. Basic information of the top $2 \%$ genes with high UMI in each tissue. Table S53. List of marker information (top 50) for each subpopulation of NK cells. Table S54. Suspiciously contaminated genes removed in each tissue for fibroblast, smooth muscle and FibSmo cell clustering. Table S55. Suspiciously contaminated genes removed in each tissue for T and NK cell clustering. Table S56. Suspiciously contaminated genes removed in each tissue for B and plasma cell clustering. Table S57. Suspiciously contaminated genes removed in each tissue for endothelial cell clustering. Table S58. Suspiciously contaminated genes removed in each tissue for myeloid cell clustering. Table S59. Antibodies used for immunostaining.

Additional file 12. Review history. 


\section{Acknowledgements}

We acknowledge Chunlin Luo, Xixi Chen, Xiangyu Xiong, Shuqiang Liu, Xiaochen Xu, Yaqing Zhou, Yanchun Feng, Jingjing He, and Liping Xu from SYSUCC for performing the tissue dissociation, and Cliff Y. Yang, Junchao Dong, and Jun Chen from Sun Yat-sen University Zhongshan School of Medicine for scientific discussions. We thank all staffs at the High-Throughput Analysis Platform (HTAP) of SYSUCC for data generation and processing.

\section{Review history}

The review history is available as Additional file 12.

\section{Peer review information}

Yixin Yao was the primary editor of this article and managed its editorial process and peer review in collaboration with the rest of the editorial team.

\section{Authors' contributions}

JXB, XSH, and ZYG conceived and supervised this study. SH, LHW, YL, YQL, JHX, HTC, WP, GWL, PPW, BL, XXJ, and DW performed the sample preparation, library construction, and data generation. SH, LHW, YL, and JXB performed the data analyses and annotated the results. SH, JXB, and ZYG wrote the manuscript. All authors read and approved the final manuscript.

\section{Funding}

This work was supported by grants as follows: the National Natural Science Foundation of China $(81970564,81471583$, and 81570587), the Guangdong Innovative and Entrepreneurial Research Team Program (2016ZTO6S638), Guangdong Provincial Key Laboratory of Organ Donation and Transplant Immunology, The First Affiliated Hospital, Sun Yat-sen University, Guangzhou, China (2013A061401007 and 2017B030314018), Guangdong Provincial International Cooperation Base of Science and Technology (Organ Transplantation), The First Affiliated Hospital, Sun Yat-sen University, Guangzhou, China (2015B050501002), the Guangdong Provincial Natural Science Funds for Major Basic Science Culture Project (2015A030308010), the Guangdong Provincial Natural Science Funds for Distinguished Young Scholars (2015A030306025), the Special Support Program for Training High Level Talents in Guangdong Province (2015TQ01R168), the Pearl River Nova Program of Guangzhou (201506010014), the Science and Technology Program of Guangzhou (201704020150), the National Program for Support of Top-Notch Young Professionals (J.-X.B.), the Chang Jiang Scholars Program (J.-X.B.), the Special Support Program of Guangdong (J.-X.B.), and Sun Yat-sen University's Young Teacher Key Cultivate Project (17ykzd29).

\section{Availability of data and materials}

The AHCA dataset has been deposited in Gene Expression Omnibus (GEO) repository with the primary accession code GSE159929 [91]. The key raw count matrices have been deposited in the Research Data Deposit (RDD, No: RDDB2020000820; http://www.researchdata.org.cn). For B and epithelial cells in the HCL dataset [20], we obtained the gene expression matrices excluding batch genes from the website (https://figshare.com/articles/HCL_DGE_Data/72354 71). Two skin datasets were available at the Gene Expression Omnibus (GEO; https://www.ncbi.nlm.nih.gov/geo/), with accession numbers GSE130973 [86] and GSE147424 [87]. A heart dataset was retrieved via https://singlecell. broadinstitute.org/single_cell/study/SCP498/transcriptional-and-cellular-diversity-of-the-human-heart [88]. Two FibSmo cell datasets were obtained from the websites $[20,89]$ (https://figshare.com/articles/Single-cell_transcriptomic_map_ of_the_human_and_mouse_bladders/8942663/1 and https://db.cngb.org/HCL). All related codes and data analysis scripts are available at https://github.com/bei-lab/scRNA-AHCA [92] and Zenodo (https://doi.org/10.5281/zenodo. 4136735) [93].

\section{Ethics approval and consent to participate}

Informed consent for the sample collection for single-cell RNA sequencing was obtained from the donor's family delegate. The management of organs was compiled according to the organ procurement guidelines. For samples collected for immunohistochemistry assays, informed consent was obtained from each donor's family delegate. This study was approved by the Ethics Committee for Clinical Research and Animal Trials of the First Affiliated Hospital of Sun Yat-sen University (permit no. [2018]255) and was conducted in accordance with the Declaration of Helsinki principles.

\section{Competing interests}

The authors declare no conflict of interest.

\section{Author details}

${ }^{1}$ Sun Yat-sen University Cancer Center, State Key Laboratory of Oncology in South China, Collaborative Innovation Center for Cancer Medicine, Guangzhou 510060, People's Republic of China. ${ }^{2}$ Organ Transplant Center, The First Affiliated Hospital, Sun Yat-sen University, Guangzhou 510080, People's Republic of China. ${ }^{3}$ Guangdong Provincial Key Laboratory of Organ Donation and Transplant Immunology, The First Affiliated Hospital, Sun Yat-sen University, Guangzhou 510080, People's Republic of China. ${ }^{4}$ Guangdong Provincial International Cooperation Base of Science and Technology (Organ Transplantation), The First Affiliated Hospital, Sun Yat-sen University, Guangzhou 510080, People's Republic of China. ${ }^{5}$ Department of Laboratory Medicine, Zhujiang Hospital, Southern Medical University, Guangzhou 510282, People's Republic of China. ${ }^{6}$ Department of Biochemistry and Molecular Biology, Zhongshan School of Medicine, Sun Yat-sen University, Guangzhou 510080, People's Republic of China. 'RNA Biomedical Institute, Sun Yat-sen Memorial Hospital, Sun Yat-sen University, Guangzhou 510120, People's Republic of China. ${ }^{8}$ Center for Precision Medicine, Sun Yat-sen University, Guangzhou 510080, People's Republic of China. 
Received: 10 March 2020 Accepted: 20 November 2020

Published online: 07 December 2020

\section{References}

1. Dalerba P, Kalisky T Jr, Sahoo D, Rajendran PS, Rothenberg ME, Leyrat AA, Sim S, Okamoto J, Johnston DM, Qian D, et al: Single-cell dissection of transcriptional heterogeneity in human colon tumors. Nat Biotechnol 2011, 29:1120-1127.

2. Klein AM, Mazutis L, Akartuna I, Tallapragada N, Veres A, Li V, Peshkin L, Weitz DA, Kirschner MW. Droplet barcoding for single-cell transcriptomics applied to embryonic stem cells. Cell. 2015;161:1187-201.

3. Wu AR, Neff NF, Kalisky T, Dalerba P, Treutlein B, Rothenberg ME, Mburu FM, Mantalas GL, Sim S, Clarke MF, Quake SR. Quantitative assessment of single-cell RNA-sequencing methods. Nat Methods. 2014;11:41-6.

4. Qiu X, Hill A, Packer J, Lin D, Ma YA, Trapnell C. Single-cell mRNA quantification and differential analysis with Census. Nat Methods. 2017;14:309-15.

5. Fabre PJ, Leleu M, Mascrez B, Lo Giudice Q, Cobb J, Duboule D. Heterogeneous combinatorial expression of Hoxd genes in single cells during limb development. BMC Biol. 2018;16:101.

6. Butler A, Hoffman P, Smibert P, Papalexi E, Satija R. Integrating single-cell transcriptomic data across different conditions, technologies, and species. Nat Biotechnol. 2018;36:411-20.

7. Haghverdi L, Lun ATL, Morgan MD, Marioni JC. Batch effects in single-cell RNA-sequencing data are corrected by matching mutual nearest neighbors. Nat Biotechnol. 2018;36:421-7.

8. Tabula Muris C, Overall c, Logistical c, Organ c, processing, Library p, sequencing, Computational data a, Cell type a, Writing g, et al. Single-cell transcriptomics of 20 mouse organs creates a Tabula Muris. Nature. 2018;562:367-72.

9. Han X, Wang R, Zhou Y, Fei L, Sun H, Lai S, Saadatpour A, Zhou Z, Chen H, Ye F, et al. Mapping the mouse cell atlas by microwell-seq. Cell. 2018;173:1307.

10. Wagner DE, Weinreb C, Collins ZM, Briggs JA, Megason SG, Klein AM. Single-cell mapping of gene expression landscapes and lineage in the zebrafish embryo. Science. 2018;360:981-7.

11. Briggs JA, Weinreb C, Wagner DE, Megason S, Peshkin L, Kirschner MW, Klein AM. The dynamics of gene expression in vertebrate embryogenesis at single-cell resolution. Science. 2018;360(6392):eaar5780.

12. Rosenberg AB, Roco CM, Muscat RA, Kuchina A, Sample P, Yao Z, Graybuck LT, Peeler DJ, Mukherjee S, Chen W, et al. Single-cell profiling of the developing mouse brain and spinal cord with split-pool barcoding. Science. 2018;360:176-82.

13. Young MD, Mitchell TJ, Vieira Braga FA, Tran MGB, Stewart BJ, Ferdinand JR, Collord G, Botting RA, Popescu DM, Loudon KW, et al. Single-cell transcriptomes from human kidneys reveal the cellular identity of renal tumors. Science. 2018;361: 594-9.

14. Schiller HB, Montoro DT, Simon LM, Rawlins EL, Meyer KB, Strunz M, Vieira Braga FA, Timens W, Koppelman GH, Budinger GRS, et al. The human lung cell atlas: a high-resolution reference map of the human lung in health and disease. Am J Respir Cell Mol Biol. 2019;61:31-41.

15. Cheng JB, Sedgewick AJ, Finnegan Al, Harirchian P, Lee J, Kwon S, Fassett MS, Golovato J, Gray M, Ghadially R, et al. Transcriptional programming of normal and inflamed human epidermis at single-cell resolution. Cell Rep. 2018;25:871-83.

16. Plasschaert LW, Zilionis R, Choo-Wing R, Savova V, Knehr J, Roma G, Klein AM, Jaffe AB. A single-cell atlas of the airway epithelium reveals the CFTR-rich pulmonary ionocyte. Nature. 2018;560:377-81.

17. Giladi A, Paul F, Herzog Y, Lubling Y, Weiner A, Yofe I, Jaitin D, Cabezas-Wallscheid N, Dress R, Ginhoux F, et al. Singlecell characterization of haematopoietic progenitors and their trajectories in homeostasis and perturbed haematopoiesis. Nat Cell Biol. 2018;20:836-46.

18. Vento-Tormo R, Efremova M, Botting RA, Turco MY, Vento-Tormo M, Meyer KB, Park JE, Stephenson E, Polanski K, Goncalves A, et al. Single-cell reconstruction of the early maternal-fetal interface in humans. Nature. 2018;563:347-53.

19. Cao J, Spielmann M, Qiu X, Huang X, Ibrahim DM, Hill AJ, Zhang F, Mundlos S, Christiansen L, Steemers FJ, et al. The single-cell transcriptional landscape of mammalian organogenesis. Nature. 2019;566:496-502.

20. Han X, Zhou Z, Fei L, Sun H, Wang R, Chen Y, Chen H, Wang J, Tang H, Ge W, et al. Construction of a human cell landscape at single-cell level. Nature. 2020;581:303-9.

21. Mizumoto N, Takashima A. CD1a and langerin: acting as more than Langerhans cell markers. J Clin Invest. 2004;113:658-60.

22. Wong MT, Ong DE, Lim FS, Teng KW, McGovern N, Narayanan S, Ho WQ, Cerny D, Tan HK, Anicete R, et al. A highdimensional atlas of human T cell diversity reveals tissue-specific trafficking and cytokine signatures. Immunity. 2016;45: 442-56.

23. Zhang L, Yu X, Zheng L, Zhang Y, Li Y, Fang Q, Gao R, Kang B, Zhang Q, Huang JY, et al. Lineage tracking reveals dynamic relationships of T cells in colorectal cancer. Nature. 2018;564:268-72.

24. Rothenberg EV. The chromatin landscape and transcription factors in T cell programming. Trends Immunol. 2014;35: 195-204.

25. Miragaia RJ, Gomes T, Chomka A, Jardine L, Riedel A, Hegazy AN, Whibley N, Tucci A, Chen X, Lindeman I, et al. Singlecell transcriptomics of regulatory T cells reveals trajectories of tissue adaptation. Immunity. 2019:50:493-504 e497.

26. Willinger T, Freeman T, Herbert M, Hasegawa H, McMichael AJ, Callan MF. Human naive CD8 T cells down-regulate expression of the WNT pathway transcription factors lymphoid enhancer binding factor 1 and transcription factor 7 ( $T$ cell factor-1) following antigen encounter in vitro and in vivo. J Immunol. 2006;176:1439-46.

27. Sebzda E, Zou Z, Lee JS, Wang T, Kahn ML. Transcription factor KLF2 regulates the migration of naive T cells by restricting chemokine receptor expression patterns. Nat Immunol. 2008;9:292-300.

28. Wang D, Diao H, Getzler AJ, Rogal W, Frederick MA, Milner J, Yu B, Crotty S, Goldrath AW, Pipkin ME. The transcription factor Runx3 establishes chromatin accessibility of cis-regulatory landscapes that drive memory cytotoxic T lymphocyte formation. Immunity. 2018;48:659-674.e656.

29. Kano S, Sato K, Morishita Y, Vollstedt S, Kim S, Bishop K, Honda K, Kubo M, Taniguchi T. The contribution of transcription factor IRF1 to the interferon-gamma-interleukin 12 signaling axis and TH1 versus TH-17 differentiation of CD4+ T cells. Nat Immunol. 2008;9:34-41.

30. Agematsu K, Hokibara S, Nagumo H, Komiyama A. CD27: a memory B-cell marker. Immunol Today. 2000;21:204-6.

31. Herling M, Patel KA, Weit N, Lilienthal N, Hallek M, Keating MJ, Jones D. High TCL1 levels are a marker of B-cell receptor pathway responsiveness and adverse outcome in chronic lymphocytic leukemia. Blood. 2009;114:4675-86. 
32. Yoon HS, Scharer CD, Majumder P, Davis CW, Butler R, Zinzow-Kramer W, Skountzou I, Koutsonanos DG, Ahmed R, Boss JM. ZBTB32 is an early repressor of the CIITA and MHC class II gene expression during B cell differentiation to plasma cells. J Immunol (Baltimore). 2012;189:2393-403.

33. Shinnakasu R, Kurosaki T. Regulation of memory B and plasma cell differentiation. Curr Opin Immunol. 2017;45:126-31.

34. Fernandez D, Ortiz M, Rodriguez L, Garcia A, Martinez D, Moreno de Alboran I. The proto-oncogene c-myc regulates antibody secretion and Ig class switch recombination. J Immunol. 2013;190:6135-44.

35. Roy K, Mitchell S, Liu Y, Ohta S, Lin YS, Metzig MO, Nutt SL, Hoffmann A. A regulatory circuit controlling the dynamics of NFkappaB cRel transitions B cells from proliferation to plasma cell differentiation. Immunity. 2019;50:616-28 e616.

36. Hashimoto D, Chow A, Noizat C, Teo P, Beasley MB, Leboeuf M, Becker CD, See P, Price J, Lucas D, et al. Tissue-resident macrophages self-maintain locally throughout adult life with minimal contribution from circulating monocytes. Immunity. 2013;38:792-804.

37. Ginhoux F, Guilliams M. Tissue-resident macrophage ontogeny and homeostasis. Immunity. 2016;44:439-49.

38. Davies LC, Jenkins SJ, Allen JE, Taylor PR. Tissue-resident macrophages. Nat Immunol. 2013;14:986-95.

39. Gao S, Yan L, Wang R, Li J, Yong J, Zhou X, Wei Y, Wu X, Wang X, Fan X, et al. Tracing the temporal-spatial transcriptome landscapes of the human fetal digestive tract using single-cell RNA-sequencing. Nat Cell Biol. 2018;20:721-34.

40. Valledor AF, Borràs FE, Cullell-Young M, Celada A. Transcription factors that regulate monocyte/macrophage differentiation. J Leukoc Biol. 1998;63:405-17.

41. Kueh HY, Champhekar A, Champhekhar A, Nutt SL, Elowitz MB, Rothenberg EV. Positive feedback between PU.1 and the cell cycle controls myeloid differentiation. Science. 2013;341:670-3.

42. Roche PA, Furuta K. The ins and outs of MHC class II-mediated antigen processing and presentation. Nat Rev Immunol. 2015;15:203-16.

43. Amatschek S, Kriehuber E, Bauer W, Reininger B, Meraner P, Wolpl A, Schweifer N, Haslinger C, Stingl G, Maurer D. Blood and lymphatic endothelial cell-specific differentiation programs are stringently controlled by the tissue environment. Blood. 2007;109:4777-85.

44. Mueller SN, Germain RN. Stromal cell contributions to the homeostasis and functionality of the immune system. Nat Rev Immunol. 2009:9:618-29.

45. Carmeliet P, Schoonjans L, Kieckens L, Ream B, Degen J, Bronson R, De Vos R, van den Oord JJ, Collen D, Mulligan RC. Physiological consequences of loss of plasminogen activator gene function in mice. Nature. 1994;368:419-24.

46. Volpert OV, Pili R, Sikder HA, Nelius T, Zaichuk T, Morris C, Shiflett CB, Devlin MK, Conant K, Alani RM. Id1 regulates angiogenesis through transcriptional repression of thrombospondin-1. Cancer Cell. 2002;2:473-83.

47. Crane NJ, Brown TS, Evans KN, Hawksworth JS, Hussey S, Tadaki DK, Elster EA. Monitoring the healing of combat wounds using Raman spectroscopic mapping. Wound Repair Regen. 2010;18:409-16.

48. Efremova M, Vento-Tormo M, Teichmann SA, Vento-Tormo R. CellPhoneDB: inferring cell-cell communication from combined expression of multi-subunit ligand-receptor complexes. Nat Protoc. 2020;15:1484-506.

49. Orian-Rousseau V. CD44 acts as a signaling platform controlling tumor progression and metastasis. Front Immunol. 2015;6:154.

50. Calame DG, Mueller-Ortiz SL, Morales JE, Wetsel RA. The C5a anaphylatoxin receptor (C5aR1) protects against Listeria monocytogenes infection by inhibiting type 1 IFN expression. J Immunol. 2014;193:5099-107.

51. Stein R, Mattes MJ, Cardillo TM, Hansen HJ, Chang CH, Burton J, Govindan S, Goldenberg DM. CD74: a new candidate target for the immunotherapy of B-cell neoplasms. Clin Cancer Res. 2007;13:5556s-63s.

52. Osei-Owusu P, Charlton TM, Kim HK, Missiakas D, Schneewind O. FPR1 is the plague receptor on host immune cells. Nature. 2019;574:57-62.

53. Contento RL, Molon B, Boularan C, Pozzan T, Manes S, Marullo S, Viola A. CXCR4-CCR5: a couple modulating T cell functions. Proc Natl Acad Sci U S A. 2008;105:10101-6.

54. Thome JJ, Yudanin N, Ohmura Y, Kubota M, Grinshpun B, Sathaliyawala T, Kato T, Lerner H, Shen Y, Farber DL. Spatial map of human T cell compartmentalization and maintenance over decades of life. Cell. 2014;159:814-28.

55. Farber DL, Yudanin NA, Restifo NP. Human memory T cells: generation, compartmentalization and homeostasis. Nat Rev Immunol. 2014;14:24-35.

56. Casey KA, Fraser KA, Schenkel JM, Moran A, Abt MC, Beura LK, Lucas PJ, Artis D, Wherry EJ, Hogquist K, et al. Antigenindependent differentiation and maintenance of effector-like resident memory T cells in tissues. J Immunol. 2012;188:4866-75.

57. Cheroutre H. IELs: enforcing law and order in the court of the intestinal epithelium. Immunol Rev. 2005;206:114-31.

58. Lees JR, Farber DL. Generation, persistence and plasticity of CD4 T-cell memories. Immunology. 2010;130:463-70.

59. Behr FM, Chuwonpad A, Stark R, van Gisbergen K. Armed and ready: transcriptional regulation of tissue-resident memory CD8 T cells. Front Immunol. 2018;9:1770.

60. Kurd NS, He Z, Milner JJ, Omilusik KD, Louis TL, Tsai MS, Widjaja CE, Kanbar JN, Olvera JG, Tysl T, et al: Molecular determinants and heterogeneity of tissue-resident memory $\mathrm{CD}^{+}{ }^{+} \mathrm{T}$ lymphocytes revealed by single-cell RNA sequencing. bioRxiv. 2020:2020.03.02.973578.

61. Fonseca R, Beura LK, Quarnstrom CF, Ghoneim HE, Fan Y, Zebley CC, Scott MC, Fares-Frederickson NJ, Wijeyesinghe S, Thompson EA, et al. Developmental plasticity allows outside-in immune responses by resident memory T cells. Nat Immunol. 2020;21:412-21.

62. Schenkel JM, Masopust D. Tissue-resident memory T cells. Immunity. 2014;41:886-97.

63. Cano-Gamez E, Soskic B, Roumeliotis TI, So E, Smyth DJ, Baldrighi M, Wille D, Nakic N, Esparza-Gordillo J, Larminie CGC, et al. Single-cell transcriptomics identifies an effectorness gradient shaping the response of CD4(+) T cells to cytokines. Nat Commun. 2020;11:1801.

64. Zhang X, Chang Li X, Xiao X, Sun R, Tian Z, Wei H. CD4(+)CD62L(+) central memory T cells can be converted to Foxp3(+) T cells. Plos One. 2013;8:e77322.

65. Qian BZ, Li J, Zhang H, Kitamura T, Zhang J, Campion LR, Kaiser EA, Snyder LA, Pollard JW. CCL2 recruits inflammatory monocytes to facilitate breast-tumour metastasis. Nature. 2011;475:222-5.

66. Yona S, Kim KW, Wolf Y, Mildner A, Varol D, Breker M, Strauss-Ayali D, Viukov S, Guilliams M, Misharin A, et al. Fate mapping reveals origins and dynamics of monocytes and tissue macrophages under homeostasis. Immunity. 2013;38: 79-91. 
67. Hume DA, Ross IL, Himes SR, Sasmono RT, Wells CA, Ravasi T. The mononuclear phagocyte system revisited. J Leukoc Biol. 2002;72:621-7.

68. Hume DA. The mononuclear phagocyte system. Curr Opin Immunol. 2006;18:49-53.

69. Jakubzick C, Gautier EL, Gibbings SL, Sojka DK, Schlitzer A, Johnson TE, Ivanov S, Duan Q, Bala S, Condon T, et al. Minimal differentiation of classical monocytes as they survey steady-state tissues and transport antigen to lymph nodes. Immunity. 2013;39:599-610.

70. Bryant DM, Mostov KE. From cells to organs: building polarized tissue. Nat Rev Mol Cell Biol. 2008;9:887-901.

71. Macara IG, Guyer R, Richardson G, Huo Y, Ahmed SM. Epithelial homeostasis. Curr Biol. 2014;24:R815-25.

72. Lynch MD, Watt FM. Fibroblast heterogeneity: implications for human disease. J Clin Invest. 2018;128:26-35.

73. Eckers A, Haendeler J. Endothelial cells in health and disease. Antioxid Redox Signal. 2015;22:1209-11.

74. Haber AL, Biton M, Rogel N, Herbst RH, Shekhar K, Smillie C, Burgin G, Delorey TM, Howitt MR, Katz Y, et al. A single-cell survey of the small intestinal epithelium. Nature. 2017;551:333-9.

75. Nguyen QH, Pervolarakis N, Blake K, Ma D, Davis RT, James N, Phung AT, Willey E, Kumar R, Jabart E, et al. Profiling human breast epithelial cells using single cell RNA sequencing identifies cell diversity. Nat Commun. 2018;9:2028.

76. Dong J, Hu Y, Fan X, Wu X, Mao Y, Hu B, Guo H, Wen L, Tang F. Single-cell RNA-seq analysis unveils a prevalent epithelial/mesenchymal hybrid state during mouse organogenesis. Genome Biol. 2018;19:31.

77. Zorn AM, Wells JM. Vertebrate endoderm development and organ formation. Annu Rev Cell Dev Biol. 2009;25:221-51.

78. Gunther J, Seyfert HM. The first line of defence: insights into mechanisms and relevance of phagocytosis in epithelial cells. Semin Immunopathol. 2018;40:555-65.

79. Lee TI, Young RA. Transcriptional regulation and its misregulation in disease. Cell. 2013;152:1237-51.

80. Singh H, Khan AA, Dinner AR. Gene regulatory networks in the immune system. Trends Immunol. 2014;35:211-8.

81. van den Brink SC, Sage F, Vertesy A, Spanjaard B, Peterson-Maduro J, Baron CS, Robin C, van Oudenaarden A. Single-cell sequencing reveals dissociation-induced gene expression in tissue subpopulations. Nat Methods. 2017;14:935-6.

82. Harris RM, Kao HY, Alarcon JM, Hofmann HA, Fenton AA. Hippocampal transcriptomic responses to enzyme-mediated cellular dissociation. Hippocampus. 2019;29:876-82.

83. Dobin A, Davis CA, Schlesinger F, Drenkow J, Zaleski C, Jha S, Batut P, Chaisson M, Gingeras TR. STAR: ultrafast universal RNA-seq aligner. Bioinformatics. 2013;29:15-21.

84. Zhou Y, Zhou B, Pache L, Chang M, Khodabakhshi AH, Tanaseichuk O, Benner C, Chanda SK. Metascape provides a biologist-oriented resource for the analysis of systems-level datasets. Nat Commun. 2019;10:1523.

85. Aibar S, Gonzalez-Blas CB, Moerman T, Huynh-Thu VA, Imrichova H, Hulselmans G, Rambow F, Marine JC, Geurts P, Aerts J, et al. SCENIC: single-cell regulatory network inference and clustering. Nat Methods. 2017;14:1083-6.

86. Solé-Boldo L, Raddatz G, Schütz S, Mallm J-P, Rippe K, Lonsdorf AS, Rodríguez-Paredes M, Lyko F. Single-cell transcriptomes of the human skin reveal age-related loss of fibroblast priming. Commun Biol. 2020;3:188.

87. He H, Suryawanshi H, Morozov P, Gay-Mimbrera J, Del Duca E, Kim HJ, Kameyama N, Estrada Y, Der E, Krueger JG, et al. Single-cell transcriptome analysis of human skin identifies novel fibroblast subpopulation and enrichment of immune subsets in atopic dermatitis. J Allergy Clin Immunol. 2020;145:1615-28.

88. Tucker NR, Chaffin M, Fleming SJ, Hall AW, Parsons VA, Bedi KC Jr, Akkad AD, Herndon CN, Arduini A, Papangeli I, et al. Transcriptional and cellular diversity of the human heart. Circulation. 2020;142:466-82.

89. Yu Z, Liao J, Chen Y, Zou C, Zhang H, Cheng J, Liu D, Li T, Zhang Q, Li J, et al. Single-cell transcriptomic map of the human and mouse bladders. J Am Soc Nephrol. 2019;30:2159-76.

90. Gaude E, Frezza C. Tissue-specific and convergent metabolic transformation of cancer correlates with metastatic potential and patient survival. Nat Commun. 2016;7:13041.

91. He S, Wang L-H, Liu Y, Li Y-Q, Chen H-T, Xu J-H, Peng W, Lin G-W, Wei P-P, Li B, Xia X, Wang D, Bei J-X, He X, Guo Z. Single-cell transcriptome profiling of an adult human cell atlas of 15 major organs. Datasets. Gene Expression Omnibus. 2020. https://www.ncbi.nlm.nih.gov/geo/query/acc.cgi?acc=GSE159929. Accessed 9 Nov 2020.

92. He S, Wang L-H, Liu Y, Li Y-Q, Chen H-T, Xu J-H, Peng W, Lin G-W, Wei P-P, Li B, Xia X, Wang D, Bei J-X, He X, Guo Z. Single-cell transcriptome profiling of an adult human cell atlas of 15 major organs. GitHub. 2020; https://github.com/ bei-lab/scRNA-AHCA. Accessed 15 July 2020.

93. He S, Wang L-H, Liu Y, Li Y-Q, Chen H-T, Xu J-H, Peng W, Lin G-W, Wei P-P, Li B, Xia X, Wang D, Bei J-X, He X, Guo Z. Single-cell transcriptome profiling of an adult human cell atlas of 15 major organs. Zenodo. 2020. https://doi.org/10. 5281/zenodo.4136735.

\section{Publisher's Note}

Springer Nature remains neutral with regard to jurisdictional claims in published maps and institutional affiliations.

Ready to submit your research? Choose BMC and benefit from:
- fast, convenient online submission
- thorough peer review by experienced researchers in your field
- rapid publication on acceptance
- support for research data, including large and complex data types
- gold Open Access which fosters wider collaboration and increased citations
- maximum visibility for your research: over 100M website views per year
At BMC, research is always in progress.
Learn more biomedcentral.com/submissions

